\title{
Short Landing Performance and Scale Effect of a Flapping Wing Aircraft
}

\author{
Si Chen ${ }^{1}$, Shijun $\mathrm{Guo}^{2}$, Hao $\mathrm{Li}^{3}$, Mingbo Tong ${ }^{4}$ and Bing $\mathrm{Ji}^{5}$ \\ ${ }^{1} \mathrm{PhD}$ Student, College of Aerospace Engineering, Nanjing University of Aeronautics and \\ Astronautics, Nanjing, P.R.China. E-mail: $406922351 @$ qq.com \\ ${ }^{2}$ Professor, School of Aerospace, Transport and Manufacturing, Cranfield University, Cranfield, \\ UK (corresponding author). E-mail: s.guo@ cranfield.ac.uk \\ ${ }^{3} \mathrm{PhD}$ student, School of Aerospace, Transport and Manufacturing, Cranfield University \\ Cranfield, UK. E-mail: $\underline{\text { H.Li@qub.ac.uk }}$ \\ ${ }^{4}$ Professor, College of Aerospace Engineering, Nanjing University of Aeronautics and \\ Astronautics, Nanjing, P.R.China. E-mail: tongw@ nuaa.edu.cn \\ ${ }^{5}$ Associate Professor, School of Control Science and Engineering, Shandong University, Jinan, \\ P.R.China. E-mail: b.ji@email.sdu.edu.cn
}

\begin{abstract}
.
An investigation has been made into the performance and scale effect of bird-like flapping wing aircraft in short landing. A flapping mechanism is proposed to transform a powered shaft rotation to an optimal kinematics of wing motion combining up-and-down stroke, pitching, fore-andback swing. An unsteady aerodynamic method (UAM) has been developed based on potential flow theory including the leading and trailing edge vortices generated by a flapping wing. After validation based on CFD results, the method is used to calculate the aerodynamic forces of flapping wings. The flight dynamics model of the aircraft is built by employing software ADAMS interfacing with the UAM coded in Python. The coupling between the inertia force of the body motion and the aerodynamic forces from flapping wing and tail-plane has been taken into the numerical simulation of the aircraft landing. Taking a bird-like aircraft model of $0.196 \mathrm{~kg}$ with a prescribed kinematics of flapping wing motion as example, parametric study has been carried out in a small range of initial tail-plane angles and subsequent flapping frequencies. Optimal parameters have been obtained to reduce the forward and descending velocities of the aircraft to a minimum value for safe and short landing performance. The study is then extended to aircraft of different geometric scale in a range of $0.5 \sim 10$ associated with weight scale $0.1 \sim 1000$. From the study, a method is developed to determine the required flapping frequency for bird-like aircraft of different scale to achieve a short landing target with the descending velocity reduced to a specified value. For the above example aircraft (geometric scale 1), the flapping frequency is $4 \mathrm{~Hz}$ to reduce both descending and forward velocities to $50 \%$ of the landing performance in fixed-wing mode. While a bird-like aircraft of geometric scale 10 and landing weight $196 \mathrm{~kg}$ requires a minimum $1.25 \mathrm{~Hz}$ flapping frequency to achieve $50 \%$ reduction of the descending and forward velocities compared with the same aircraft landing in fixed-wing mode.
\end{abstract}

Keywords: Flapping wing, unsteady aerodynamic method, short landing, descending velocity

\section{Introduction}


For aircraft as well as flying animals, landing is the most tricky and critical process of a safe flight. In the past decades, huge effort in aerospace has been made to develop high lift devices such as slat, flaps and morphing wing technology to reduce aircraft landing distance, hence the demand for airport infrastructure. Research attention has also been paid to developing bird-like flapping wing unmanned aerial vehicles (UAV) and manned aircraft capable of Vertical/Short-Take-Off-and-Landing (V/STOL) and also high performance in cruise flight. A small or micro UAV with VTOL/STOL capability is suitable for carrying out missions such as Intelligence, Surveillance and Reconnaissance (ISR) in complex environment or high risky scenario. With adequate power, VTOL can be achieved normally by tilt thrust forces as studied by Martin and Tung (Martin and Tung 2004) who designed a 0.254m ducted rotor UAV. Wind tunnel test was carried out to evaluate the UAV performance in a range of tilt angles. Following the study, Akturk et al (Akturk et al. 2009) employed a planar particle image velocimeter (PIV) system to measure the flow field and a computational method to predict the aerodynamics around the duct in hover and forward flight condition. The study shown that the mean flow results near the fan inlet plane by experimental and computational method were in very good agreement in hover condition. Prabu et al (Prabu et al. 2016) and (Patra et al. 2017) have also made effort to achieve VTOL for a deltawing quadcopter and tilt-rotor aircraft design. Regarding the STOL capability, Englar et al (Englar et al. 1981) evaluated the lift increase and STOL capability by tangential blowing over the wing rounded trailing edge and designed a Demonstrator Aircraft. Montanya and Marshall(Montanya and Marshall 2007) also found that STOL can be realized by ejecting a tangential jet from a slot located near the airfoil trailing edge to gain effective camber and increase lift. In their research, the shortest landing distance was calculated to be $610 \mathrm{~m}$ for a flap deflection angle of ninety degrees and a blowing coefficient of 0.34. Moore et al (Moore et al. 2014) studied a post-stall perching process of a fixed-wing glider, which flies at $7 \mathrm{~m} / \mathrm{s}$ before pitching up at large angle of attack by deflecting the tail-plane for short landing.

Apart from the fixed-wing aircraft, flying animals demonstrate extraordinary capability of STOL using their flapping wings. During landing, birds will flap their wings with large amplitude and at increased frequency for several flapping cycles to create rapid deceleration and maneuvering moments to adjust their flight speed and body position relative to the perch. In this process, the angle of attack (AoA) of their wings can easily reach above $50^{\circ}$ (Paranjape et al. 2013), where the airflow won't remain attached on the wing surface (Ghosh et al. 2012). Instead, flow separation from the leading and trailing edges causes a low pressure vortex region on the wing upper surface and create an instantaneous high pressure drag. Berg and Biewener (Berg and Biewener 2008) studied the kinematics of ascending and descending flight of pigeon by high-speed video and observed that the stroke plane angle became closer to horizontal for steeper flight state (ascent or descent). The authors also estimated the power 
requirement for level flight, ascent and decent. It was found that for steep descent, greater power was required than the sum of power for level flight and the change of potential energy. Roderick et al (Roderick et al. 2017) made a survey and research on the bimodal animal locomotion and existing aerial robotics' landing, surface locomotion and take-off, and found that the animals' pitch-up landing maneuvers provide a guidance for a flapping wing design. By flapping wings, birds manipulate the unsteady aerodynamic phenomenon to create instantaneous high maneuvering force which can reduce the flight speed during the perching process. Inspired by bird flight performance, numerous design of flapping wing aerial vehicles have been proposed (Deng et al. 2014; Mishra et al. 2015; Palmer et al. 2013; Regan et al. 2002; Yang et al. 2018). Krashanitsa et al (Krashanitsa et al. 2009) built a 74-cmwing-span ornithopter equipped with an automatic flight control system and demonstrate the ornithopter's stability in all axes by flight test. Jackowski (Jackowski 2009) developed a large scale ornithopter carrying a computer and sensor package (400 gram) with a simple PD controller to realize pitch stability. Guo et al (Guo et al. 2012) designed a jumping mechanism for flapping wing driven by shape memory alloy and built the corresponding control system. Through this mechanism, they realized the takeoff process of flapping wing by hopping instead of throwing. Paranjape et al (Paranjape et al. 2013) designed an aerial robot inspired by birds and employed dihedral-based control to fulfill perch process on a human hand. In the last decade, achievement has been made to build smaller scale flapping wings capable of STOL/VTOL. Baligidad et al (Baligidad et al. 2017) designed a four wing flapping wing model of $175 \mathrm{~cm}$ in length and $14 \mathrm{~g}$ weight including a tail rotor to control yaw movement. Graule et al (Graule et al. 2016) designed a robotic insect and make it robustly perch on a range of material by implementing switchable electrostatic adhesion. Guo et al (Guo et al. 2018) made a flyable micro flapping wing rotor of only $2.6 \mathrm{~g}$ and achieved VTOL flight test.

Despite the above studies on bio-inspired flapping wing UAVs, the dynamics of their perching process of flapping wing aircraft has not been fully investigated. In particular, the strategy of performing an optimal short landing by flapping wing is yet to be well understood. The current study is focused on reducing the forward and descending velocity of different scale aircraft to achieve short landing by manipulating the tail-plane deflection, the timing of flapping motion and the flapping frequency.

The current study is presented as follow: the $2^{\text {nd }}$ section introduced a flapping wing mechanism and an unsteady aerodynamic method (UAM), which was validated by comparing with CFD results produced in previous research work (Gopalakrishnan and Tafti 2009; Wang et al. 2013). The geometric and mass parameters of the flapping mechanism was presented to obtain the exclusive flapping motion. The perching process and assumptions were set before the aerodynamic modelling of the wing and tailplane for the landing performance simulation. The short landing performance was measured by the descending and forward velocity comparing with the result of a fixed wing gliding mode at the end of 
perching process. In the $3^{\text {rd }}$ section, a parametric study of a bird-like small flapping wing aircraft of $0.196 \mathrm{~kg}$ with different flapping frequency, tail-plane deflection angles and the timing to start the flapping wing motion was carried out to evaluate the short landing performance. Then, the time course variation of the pitching angles, the descending and forward velocities of the aircraft and corresponding aerodynamic forces during the perching process are presented in detail. In the $4^{\text {th }}$ section, a relationship between the aircraft scale and flapping frequency required to achieve a short landing target was established. The study result was demonstrated by a flapping wing aircraft of 10 times larger in geometry than the above small model. Finally conclusions were drawn in $5^{\text {th }}$ section.

\section{The Flapping Wing Aircraft and Theoretical Methods}

\section{The aircraft model and flapping mechanism}

In this study, the configuration of a small flapping wing aircraft model is illustrated in Error! Reference source not found.. The wing of airfoil NACA0006 is of semi-span $0.45 \mathrm{~m}$ and chord $0.11 \mathrm{~m}$. The dimensions of the model body and an all moveable tail-plane of airfoil NACA0006 are also shown in Error! Reference source not found. (similar to a small seagull).
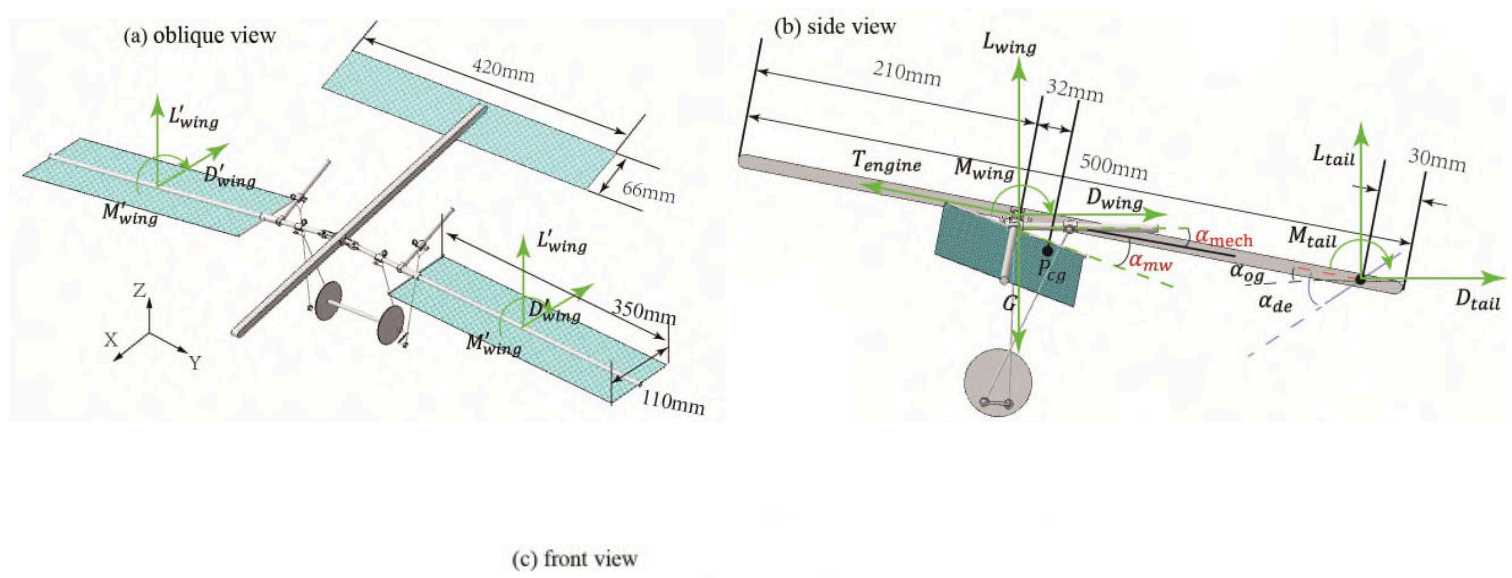

Figure 1. (a) 3D view, (b) side view and (c) front view of the aircraft model and flapping wing mechanism

The model total mass is $0.196 \mathrm{~kg}$ with its center of gravity located at $P_{c g}$. The flapping wings are connected to the fuselage body forward of $P_{c g}$. The tail-plane surface area is $36 \%$ of the flapping wing and the aerodynamic center of the tail-plane is located at $30 \mathrm{~mm}$ forward of the body tail. The $\phi_{\text {mech }}$ is the stroke angle of the mechanism, which is the same as the flapping angle $\phi_{m w}$ of the wing (positive 
above the horizontal Y-axis). The $\alpha_{m w}$ and $\alpha_{m e c h}$ are the pitching angle of the flapping wing (positive for leading edge upward) and the rotation angle of the mechanism (wing beam) relative to the model body respectively. Wherein, the $\alpha_{m w}$ differs from $\alpha_{\text {mech }}$ by a constant value. The equivalent lift ( $\mathrm{L}_{\text {wing }}^{\prime}$ ), drag $\left(\mathrm{D}_{\text {wing }}^{\prime}\right)$ and pitching moment $\left(\mathrm{M}_{\text {wing }}^{\prime}\right)$ on each single wing in the ground coordinate can be converted to the whole lift $\left(\mathrm{L}_{\text {wing }}\right)$, drag $\left(\mathrm{D}_{\text {wing }}\right)$ and pitching moment $\left(\mathrm{M}_{\text {wing }}\right)$ enforced on the symmetry plane of the aircraft.

Further details of the flapping mechanism are shown in Error! Reference source not found.. A wheel is mounted on each side of the aircraft model and powered to rotate around the center $O_{1}$ by a motor through the shaft. The wheel is connected to the wing spar at joint $P_{1}$ and $P_{3}$ through the flapping linkage of length $A_{1}$, and drive the flapping wing in angle $\phi_{\text {mech }}$ around joint $O_{2}$. The wing spar is connected with the main rib of length $A_{3}$ at $P_{5}$, which is also connected to the wheel through the pitching linkage of length $A_{2}$ at point $P_{4}$ and $P_{2}$, and a crank of span $B_{3}$ between joint $P_{1}$ and $P_{2}$. The distance from $O_{1}$ to $P_{1}$ and $P_{2}$ is $r$; the distance between $O_{2}$ and $P_{3}$ is $B_{1}$, and between $P_{3}$ and $P_{5}$ is $B_{2}$. There is an angle $\varphi$ between the two linkage joint $P_{1}$ and $P_{2}$ that determines the phase difference between the flapping and pitching motion of the wing. The origin of the X-Y-Z coordinate system is located at $O_{1}$. The $C_{\mathrm{x}}, C_{\mathrm{y}}, C_{\mathrm{z}}$ represent the coordinates of the joint $O_{2}$.

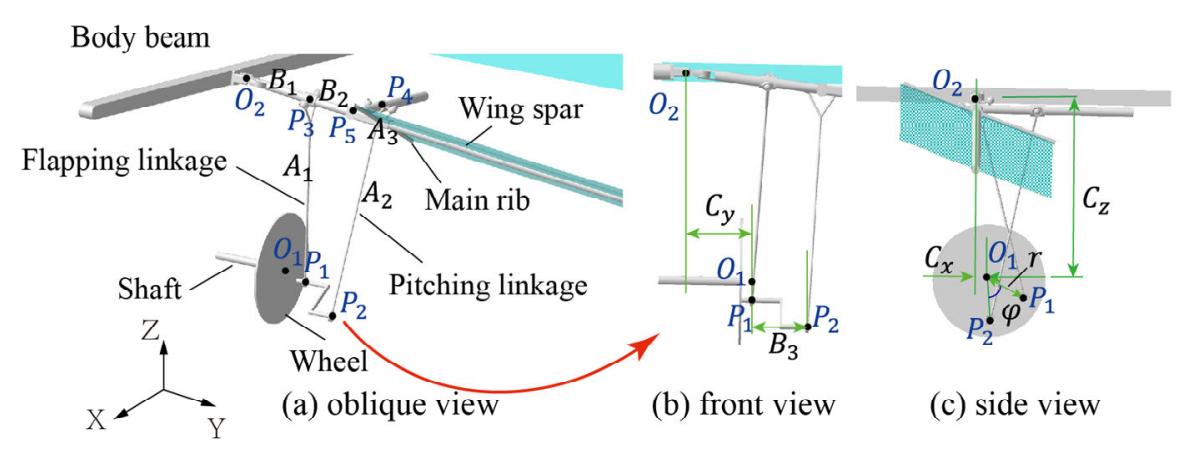

Figure 2. Geometry parameters of the components of the flapping mechanism

Based on the mechanism, a set of equations to express the flapping motion in relation to the parameters can be created as presented below. The equations can be used together with aerodynamic calculation to find an optimal kinematics of motion to obtain a specified aerodynamic efficiency or performance of a flapping wing. Alternatively, for a specified kinematics of motion, a flapping mechanism with a set of geometric parameters can be designed. For this particular case of study, the specified flapping angle, pitching angle and phase difference were set as $\phi_{\text {mech }}=-50^{\circ} \sim 50^{\circ}, \alpha_{\text {mech }}=$ $-21^{\circ} \sim 20^{\circ}$ and $\frac{3 \pi}{8}$ (measured at $\phi_{m e c h}=-50^{\circ}$ ) respectively. The requirement led to a set of 
parameters of the mechanism design as listed in Error! Reference source not found.. The mass and moment of inertia for each part of the mechanism relative to their own local center of gravity are also calculated as listed in Error! Reference source not found. for the flight dynamics model of the aircraft in landing.

Table 1. Geometric parameters for the flapping wing mechanism

\begin{tabular}{c|ccccccccccc}
\hline Parameter & $\boldsymbol{A}_{\mathbf{1}}$ & $\boldsymbol{A}_{\mathbf{2}}$ & $\boldsymbol{A}_{\mathbf{3}}$ & $\boldsymbol{B}_{\mathbf{1}}$ & $\boldsymbol{B}_{\mathbf{2}}$ & $\boldsymbol{B}_{\mathbf{3}}$ & $\boldsymbol{C}_{\boldsymbol{x}}$ & $\boldsymbol{C}_{\boldsymbol{y}}$ & $\boldsymbol{C}_{\boldsymbol{z}}$ & $\boldsymbol{r}$ & $\boldsymbol{\varphi}$ \\
\hline Dimensions $(\mathrm{mm})$ & 135 & 155 & 80 & 40 & 15 & 22 & 0 & -30 & 135 & 32 & $60(\mathrm{deg})$ \\
\hline
\end{tabular}

Table 2. Component mass and moment of inertia of the flapping wing aircraft

\begin{tabular}{cllll} 
component & Mass $[\mathbf{k g}]$ & $\boldsymbol{I}_{\boldsymbol{x x}}\left[\mathbf{k g} \cdot \mathbf{m m}^{\mathbf{2}}\right]$ & $\boldsymbol{I}_{\boldsymbol{y y}}\left[\mathbf{k g} \cdot \mathbf{m m}^{\mathbf{2}}\right]$ & $\boldsymbol{I}_{\mathbf{z z}}\left[\mathbf{k g} \cdot \mathbf{m m}^{\mathbf{2}}\right]$ \\
\hline \hline Wing spar & 0.03 & 488.0 & 0.135 & 488.0 \\
Main rib & 0.00745 & 0.0363 & 7.45 & 7.45 \\
Flapping linkage & 0.00192 & 4.36 & 4.39 & 0.0389 \\
Pitching linkage & 0.00211 & 6.22 & 6.18 & 0.0390 \\
Wheel & 0.00453 & 1.81 & 3.64 & 1.89 \\
Shaft & 0.0119 & 6.08 & 0.0373 & 6.08 \\
Body beam & 0.137 & 2.27 & 2.820 & 2820 \\
Other parts & 0.00129 & 0.0278 & 0.0161 & 0.0339 \\
\hline \hline Total landing mass & 0.196 & 2750.0 & 3580.0 & 5720.0
\end{tabular}

The mechanism transformed the powered rotation into a combination of flapping, pitching and swing motion of a flapping wing. To obtain the kinematics of motion of the flapping wing, the position of $P_{1}$, $\mathrm{O}_{2}$ and $\mathrm{P}_{2}$ varies with the rotation of the wheel in the X-Y-Z system and can be determined by:

$$
\begin{gathered}
\overrightarrow{O_{1}}=[0,0,0], \overrightarrow{O_{2}}=\left[O_{2 x}, O_{2 y}, O_{2 z}\right] \\
\overrightarrow{P_{1}}=[r * \cos (\omega * t+\varphi), r * \sin (\omega * t+\varphi), 0] \\
\overrightarrow{P_{2}}=\left[r * \cos (\omega * t), r * \sin (\omega * t), B_{4}\right]
\end{gathered}
$$

where $\omega$ is angular velocity $(\mathrm{rad} / \mathrm{s})$ of the rotating wheel.

Since the space location of $P_{3}$ can only be revolved about $O_{2}, P_{3}$ and $O_{2}$ share the same coordinate in $X$ direction. A set of equations can be derived and used to calculate the position of $P_{3}$ as follows.

$$
\begin{gathered}
\left\|\overrightarrow{P_{3}}-\overrightarrow{O_{2}}\right\|=B_{1} \\
\left\|\overrightarrow{P_{3}}-\overrightarrow{P_{1}}\right\|=A_{1} \\
\overrightarrow{P_{3}}=\left[O_{2 x}, P_{3 y}, P_{3 z}\right]
\end{gathered}
$$

From the $P_{3}$ coordinate, the $P_{5}$ and $P_{4}$ position can be determined by the following equations: 


$$
\begin{gathered}
\overrightarrow{P_{5}}=\left(B_{1}+B_{2}\right) * \frac{\overrightarrow{P_{3}}-\overrightarrow{O_{2}}}{B_{1}}+\overrightarrow{O_{2}} \\
\left\|\overrightarrow{P_{4}}-\overrightarrow{P_{5}}\right\|=A_{3} \\
\left\|\overrightarrow{P_{2}}-\overrightarrow{P_{5}}\right\|=A_{2} \\
\left(\overrightarrow{P_{4}}-\overrightarrow{P_{5}}\right) \cdot\left(\overrightarrow{P_{5}}-\overrightarrow{O_{2}}\right)=0
\end{gathered}
$$

Eq. (10) expresses that the spar between $P_{5}$ and $O_{2}$ is perpendicular to the rib between $P_{5}$ and $P_{4}$.

In this study, the above equations were coded in Python to produce a kinematics of motion of the flapping wing for aerodynamic simulation. For this case, the resulting flapping angle $\left(\phi_{\text {mech }}\right)$ and pitching angle $\left(\alpha_{\text {mech }}\right)$ of the wing spar in three flapping cycles are plotted in Error! Reference source not found.. The analysis was started at $t=0 \mathrm{~s}$ with the initial location of $P_{1}$ and $P_{2}$ as shown in Error! Reference source not found.(c). The flapping amplitude measured at $P_{3}$ was $0.065 \mathrm{~m}$ to obtain flapping angle $\phi_{\text {mech }}=-50^{\circ} \sim 50^{\circ}$. The phase difference between $\phi_{\text {mech }}$ and $\alpha_{\text {mech }}$ was $\frac{3 \pi}{8}$ (measured at the valley of the curves) and $\frac{\pi}{4}$ (measured at the peak of the curves). The maximum pitching angle $\left(20^{\circ}\right)$ in one flapping cycle occurred when $\hat{t}=0.96$ while the smallest angle $-21.8^{\circ}$ at $\hat{t}=0.37$.

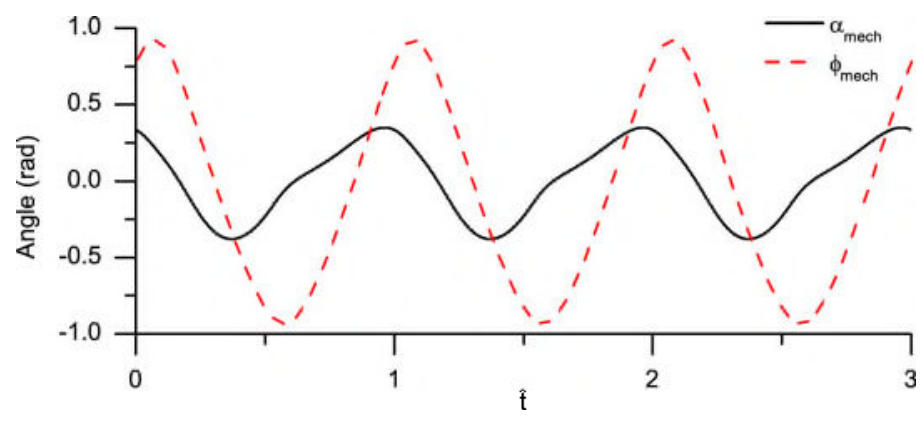

Figure 3. Flapping angle (positive when $P_{3 z}>O_{2 z}$ ) and the twist angle (positive in the nose-up direction) of the flapping wing mechanism in three flapping period. $\hat{t}$ is the non-dimensional time normalized by flapping period.

\section{The unsteady aerodynamic method}

For a 2D airfoil as illustrated in Error! Reference source not found.(a), the classical Joukowski transformation can be applied to uniquely map a circle of radius $R$ in the $z$-plane into an airfoil in the $\zeta$ plane. The transformation can be expressed in the equation as follows:

$$
\zeta=\mathrm{z}+\frac{(1-\varepsilon) R^{2}}{z}+\frac{R^{3} \varepsilon}{2 z^{2}}, \quad \varepsilon=\frac{(\tau-i \sigma)}{R}
$$


where $\zeta$ and $z$ are the complex coordinates in the $\zeta$-plane and the $z$-plane respectively; $\tau$ and $\sigma$ are non-dimensional factors governing the thickness and the camber of the airfoil (Ansari et al. 2006a). The equation (11) can be also written in $\zeta$-plane:

$$
\begin{gathered}
\xi=2 R \cos \theta+\sigma\left(\sin \theta-\frac{1}{2} \sin 2 \theta\right)-\tau\left(\cos \theta-\frac{1}{2} \cos 2 \theta\right) \\
\eta=\tau\left(\sin \theta-\frac{1}{2} \sin 2 \theta\right)+\sigma\left(\cos \theta-\frac{1}{2} \cos 2 \theta\right)
\end{gathered}
$$

where $\theta$ is the angular displacement about the origin of the $z$-plane.

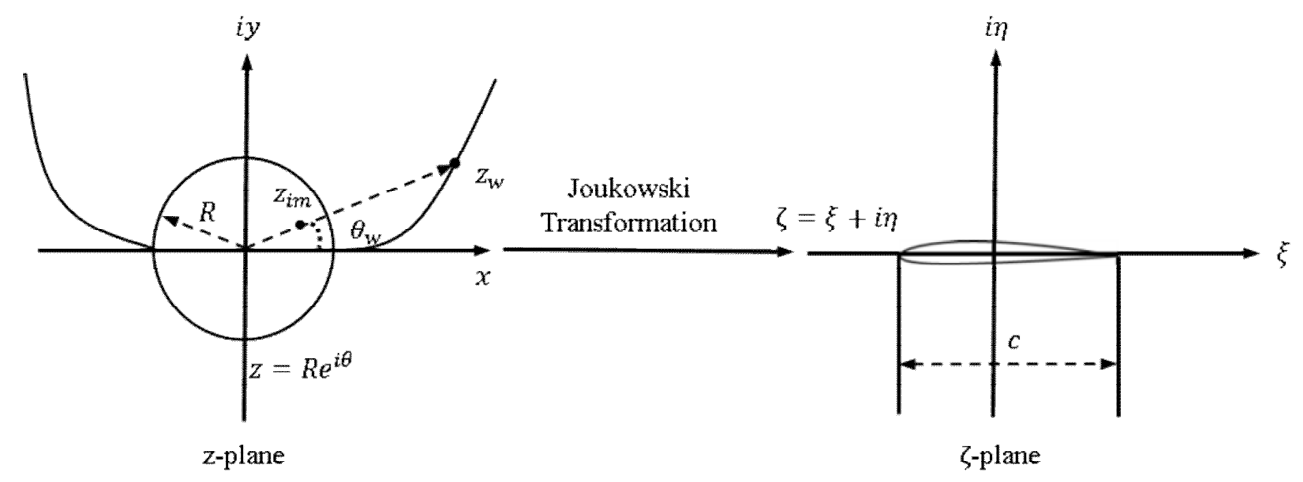

(a)

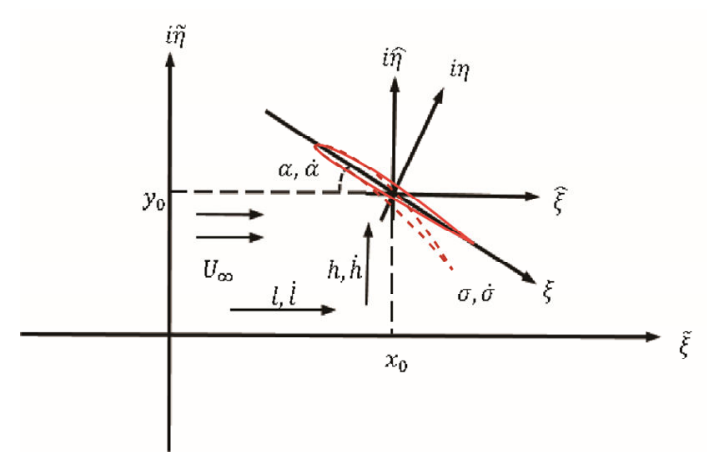

(b)

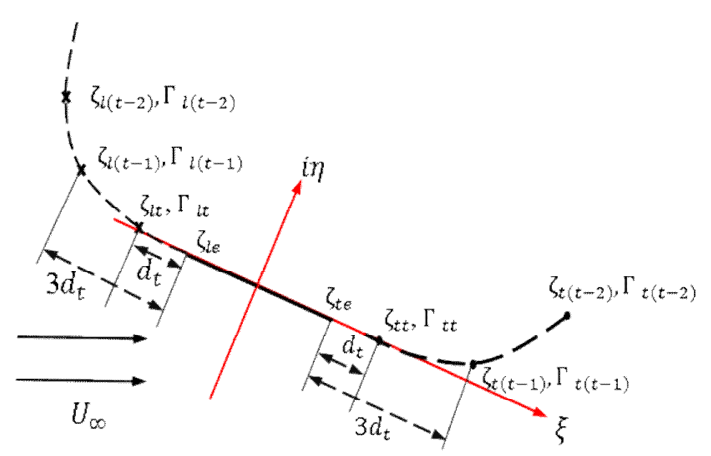

(c)

Figure 4. (a) Joukowski Transformation of 2D airfoil (b) 2D airfoil motions in the $\zeta$-plane (c) Vortex shedding model for the 2D airfoil

In this model, the flow potential could be divided into two terms (Ansari et al. 2006a): the quasisteady term and the unsteady term. The quasi-steady term is relevant to the freestream $\left(v_{\theta f}\right)$ and airfoil motion $\left(v_{\theta m}\right)$, while the unsteady term is related to the roll-up of the leading and trailing edge vortices. By enforcing the zero-through-flow boundary condition on the airfoil surface and Kutta-Joukowski condition at both trailing and leading edges of the airfoil, the distribution of total vortex sheet (density of circulation) including quasi-steady term and unsteady term can be expressed by equation (14) and 
(15) and the position of these leading edge vortices and trailing edge vortices are calculated based on the induced velocity generated by all the other vortices' in the flow field.

$$
\begin{gathered}
\gamma_{0}=v_{\theta f}+v_{\theta m} \\
=\frac{1}{R}\left(-A_{1} \cos \theta-\left(A_{2}+0.5 A_{7}\right) \cos 2 \theta+A_{3} \sin \theta+\left(A_{4}-0.5 A_{5}+0.5 A_{6}\right) \sin 2 \theta\right. \\
-A_{8} \sin \theta \cos 2 \theta+A_{9} \sin \theta \sin 2 \theta-A_{10} \cos \theta \cos 2 \theta+A_{11} \cos \theta \sin 2 \theta-0.5 A_{12} \sin 4 \theta \\
\left.+0.5 A_{13} \sin 4 \theta\right)-2 U_{\infty} \sin (\theta-\alpha)+\frac{\Gamma_{0}}{2 \pi R} \\
\gamma_{1}=-\frac{1}{2 \pi R} \int_{t v} \Re\left(\frac{Z_{t v}+R e^{i \theta}}{z_{t v}-R e^{i \theta}}\right) \gamma_{t v} d z_{t v}-\frac{1}{2 \pi R} \int_{l v} \Re\left(\frac{z_{l v}+R e^{i \theta}}{z_{l v}-R e^{i \theta}}\right) \gamma_{l v} d z_{l v}-\frac{\Gamma_{0}}{2 \pi R} \\
\Gamma_{0}=2 \pi\left[2 R\left(\left(i-U_{\infty}\right) \sin \alpha+\dot{h} \cos \alpha\right)+\dot{\alpha}\left(\frac{1}{2} \tau^{2}+\frac{1}{2} \sigma^{2}-2 R^{2}\right)+R \dot{\sigma}+0.5 \tau \dot{\sigma}\right]
\end{gathered}
$$

where $U_{\infty}$ is the freestream speed, $\dot{l}$ is the sweeping velocity and $\dot{h}$ is plunging velocity as defined in Error! Reference source not found.(a). The total quasi-steady bound circulation and the coefficients $A_{1} \sim A_{13}$ are given by:

$$
\begin{gathered}
\Gamma_{0}=2 \pi\left[2 R\left(\left(\dot{l}-U_{\infty}\right) \sin \alpha+\dot{h} \cos \alpha\right)+\dot{\alpha}\left(\frac{1}{2} \tau^{2}+\frac{1}{2} \sigma^{2}-2 R^{2}\right)+R \dot{\sigma}+0.5 \tau \dot{\sigma}\right] \\
A_{1}=-\sigma(\dot{l} \cos \alpha-\dot{h} \sin \alpha)+(2 R-\tau)(\dot{l} \sin \alpha+\dot{h} \cos \alpha) \\
A_{2}=\sigma(\dot{l} \cos \alpha-\dot{h} \sin \alpha)+\tau(\dot{l} \sin \alpha+\dot{h} \cos \alpha) \\
A_{3}=\tau(\dot{l} \cos \alpha-\dot{h} \sin \alpha)-\sigma(\dot{l} \sin \alpha+\dot{h} \cos \alpha) \\
A_{4}=-\tau(\dot{l} \cos \alpha-\dot{h} \sin \alpha)+\sigma(\dot{l} \sin \alpha+\dot{h} \cos \alpha) \\
A_{5}=-2 R \sigma \dot{\alpha}-\sigma \dot{\sigma} \\
A_{6}=2 R \sigma \dot{\alpha}-\sigma \dot{\sigma} \\
A_{7}=4 R(\tau-R) \dot{\alpha}+2 R \dot{\sigma} \\
A_{8}=R \sigma \dot{\alpha}+1.5 \sigma \dot{\sigma} \\
A_{9}=-0.5\left(\tau^{2}+\sigma^{2}+2 R \tau\right) \dot{\alpha}-(R \dot{\sigma}+0.5 \tau \dot{\sigma}) \\
A_{10}=0.5\left(\tau^{2}+\sigma^{2}-4 R \tau\right) \dot{\alpha}+0.5 \tau \dot{\sigma} \\
A_{11}=1.5 \sigma \dot{\sigma}-2 R \sigma \dot{\alpha} \\
A_{12}=-0.5 \sigma \dot{\sigma} \\
A_{13}=-0.5 \sigma \dot{\sigma}
\end{gathered}
$$

After obtaining the vortex sheet distribution, the aerodynamic forces in $\hat{\zeta}$ frame can be calculated according to Kelvin's impulse theorem (Gordon 1989).

$$
\begin{gathered}
\hat{F}=\mathrm{i} \rho \frac{d}{d t} \int_{a} \gamma_{0} \hat{\xi} d \hat{\xi}+\mathrm{i} \rho \frac{d}{d t} \int_{a} \gamma_{1} \hat{\xi} d \hat{\xi}+\mathrm{i} \rho \frac{d}{d t} \int_{a} \gamma_{t v} \hat{\xi} d \hat{\xi}+\mathrm{i} \rho \frac{d}{d t} \int_{a} \gamma_{l v} \hat{\xi} d \hat{\xi} \\
=-\Im\left(i \rho U_{0} \int_{a} \gamma_{0} \hat{\zeta} d \hat{\zeta}\right)+\frac{\rho}{2} \frac{d}{d t} \int_{a} \gamma_{0} \hat{\zeta}^{2} d \hat{\zeta}+\frac{\rho}{2} \frac{d}{d t} \int_{a} \gamma_{1} \hat{\zeta}^{2} d \hat{\zeta}-\Im\left(i \rho U_{0} \int_{a} \gamma_{1} \hat{\zeta} d \hat{\zeta}\right) \\
+\frac{\rho}{2} \frac{d}{d t} \int_{a} \gamma_{t v} \hat{\zeta}^{2} d \hat{\zeta}+\frac{\rho}{2} \frac{d}{d t} \int_{a} \gamma_{l v} \hat{\zeta}^{2} d \hat{\zeta}-\Im\left(i \rho U_{0} \int_{a} \gamma_{t v} \hat{\zeta} d \hat{\zeta}\right)-\Im\left(i \rho U_{0} \int_{a} \gamma_{l v} \hat{\zeta} d \hat{\zeta}\right)
\end{gathered}
$$


where $U_{0}=\left(-U_{\infty}+\dot{l}\right)+i \dot{h}, \gamma_{t v} d \hat{\zeta}$ is the vortex strength of the trailing edge vortex and $\gamma_{l v} d \hat{\zeta}$ is the vortex strength of the leading edge vortex.

The UAM method for a 2D wing section can be applied to a 3D wing by integrating the aerodynamic forces along the wing span. The method has been verified to ensure the result reliability in this study. The initial condition setting is kept the same as the Gopalakrishnan, P., and Tafti D.K.(Gopalakrishnan and Tafti 2009) and Wang et al(Wang et al. 2013). The geometry of the wing for comparison is a flat plate with aspect ratio $A R=4$ of a single wing and the advance ratio $\mathrm{J}=0.5$. Since $A R=4$, it is proper to set the wing semi-span as $0.4 \mathrm{~m}$ and the chord of the wing as $0.1 \mathrm{~m}$. In order to meet the requirement of Reynolds number $R e$ is $10^{4}$, the flapping frequency in this comparison case is selected to be $2 \mathrm{~Hz}$. The flapping and twisting motion is defined as equation (21) and equation (22):

$$
\begin{gathered}
\phi=\Phi \sin (2 \pi f t) \\
\alpha=\Delta \alpha \sin \left(2 \pi f t+\frac{\pi}{2}\right)+\alpha_{0}
\end{gathered}
$$

where $\phi$ is the stroke angle, $\Phi$ is the stroke amplitude, $f$ stands for flapping frequency, $t$ is the time, $\alpha$ is the pitching angle, $\Delta \alpha$ is the pitching angle amplitude and $\alpha_{0}$ is the average pitching angle during one flapping cycle.

The UAM was also coded in Python to calculate the unsteady aerodynamic forces of the flapping wing based on its kinematics of motion. To validate the UAM, the lift and thrust coefficients of a 3D flapping wing obtained by CFD method in previous study (Gopalakrishnan and Tafti 2009; Wang et al. 2013) were compared with that by UAM. As shown in Error! Reference source not found., excellent agreement of the results by the UAM and CFD method was obtained. The mean lift coefficient $\left(\overline{C_{L}}\right)$ in one flapping cycle is 0.49 by the UAM and also 0.49 by Wang et al, and 0.52 by Gopalakrishnan, P., and Tafti, D.K. with a deviation less than $6 \%$.

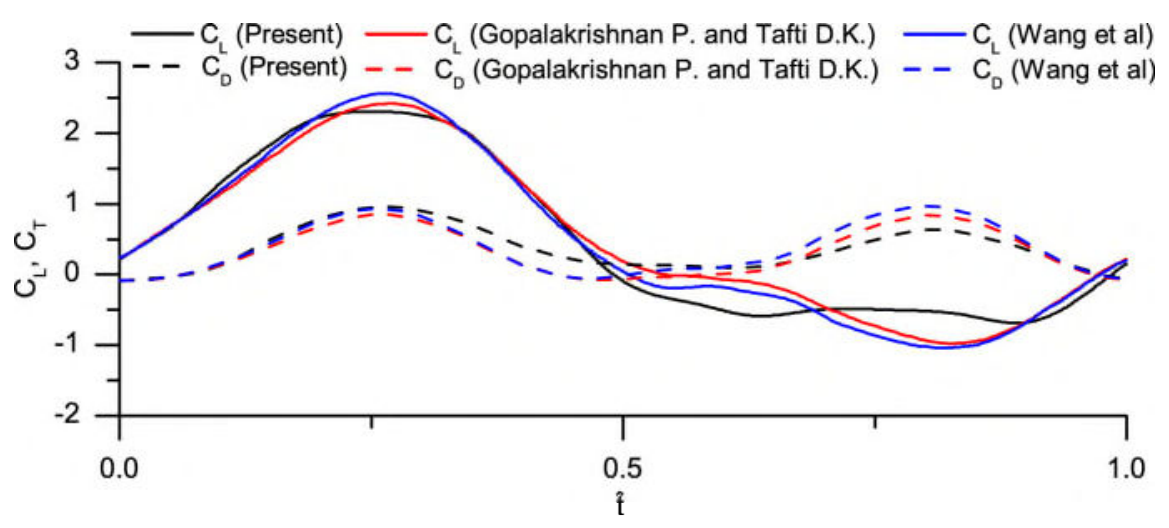


Figure 5. Comparison of the lift and thrust coefficients for validation in a case $\Phi=30^{\circ}, \Delta \alpha=32.5^{\circ}$, $\alpha_{0}=12.5^{\circ}$ and $\hat{t}$ is the non-dimensional time normalized by flapping period.

The mean thrust coefficient $\left(\overline{C_{D}}\right)$ are $0.36,0.40$ and 0.37 by the three models with a deviation between 2.7\% 10\%. The difference of the aerodynamic coefficients between UAM and CFD method is mainly caused by neglecting the spanwise flow effect in the UAM. The validation results indicate that it is appropriate to use the UAM in the present study. Additional validation cases can be also found in a previous publication (Chen et al. 2018).

To mimic the bird perching, the aircraft landing scheme is divided into three stages with the numerical simulation process shown in Error! Reference source not found.. The landing simulation was started at $t_{0}$ in level flight near ground in the stage- 1 . The aerodynamic force to keep the aircraft in steady level flight at an approaching flight speed was calculated by steady CFD method. From the stage-2 starting at $t_{d e}$, the aircraft motion was varied by an initial deflection of the tail-plane. The flight dynamics of the aircraft was modelled by using software ADAMS (Automated Dynamic Analysis of Mechanical Systems, developed by MSC Software). The UAM method was used to calculate the aerodynamic force of the wing and tail-plane, firstly a fixed wing and tail-plane deflection angle $\alpha_{\mathrm{og}}$ (positive in pitching up direction) to simulate the aircraft landing in gliding mode. The ADAMS model was coupled with the aerodynamic model by the UAM firstly through a system file by using Python code. Then, the aerodynamic lift and moment data were transferred from the system file to ADAMS model by using a user-defined "spline_read.dll" (generated by user-written "spline_read.c") coded by C language. The results provided a reference to compare with and evaluate the landing performance by flapping wings. For the flapping wing mode in stage-2, the UAM was also used to calculate the unsteady aerodynamic forces produced by the flapping wing and tail-plane coupled with the flight dynamics model in an iterative manner. The tail-plane deflection angle was increased from $\alpha_{o g}$ to a negative value (leading edge down) $\alpha_{d e}$ for a short period $d t_{d e}$ that produced a positive pitching moment, pitching angle and short upward motion of the aircraft. This was followed by the flapping wing motion in the stage-3 starting from $t_{f}$. The lift $\mathrm{L}_{\text {wing }}$ and drag $\mathrm{D}_{\text {wing }}$ produced by flapping wing together with the tailplane $\mathrm{L}_{\text {tail }}$ reduced the downward and forward velocities of the aircraft motion to achieve a short landing performance at the end $t_{\text {end }}$. 


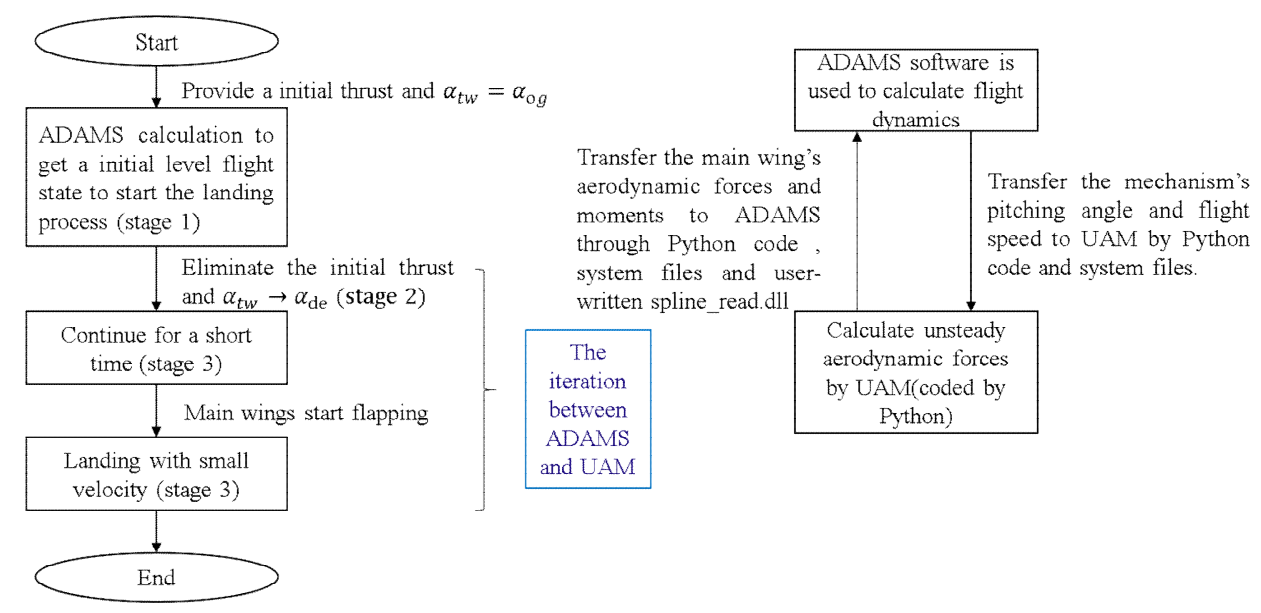

Figure 6. Flow chart of the numerical calculation process

\section{Results and Discussion}

\section{Aerodynamics of the wing and tail during level flight (landing stage-1)}

In the landing stage-1, the aerodynamic forces acting on the wings and tail-plane of the aircraft in steady level flight were calculated using CFD method (k-omega SST model in Fluent 14.5). The analysis is to determine the necessary approaching speed with an angle of attack (AoA) of the wing below 10 degree and ensure the aircraft in steady level flight condition for landing. The wing model was embedded inside a CFD model of nonstructural mesh generated by using T-grid as shown in Error! Reference source not found. to simulate the fluid field. The wing root was set in the center of the CFD model with a radius of 10 times of the wing semi-span.

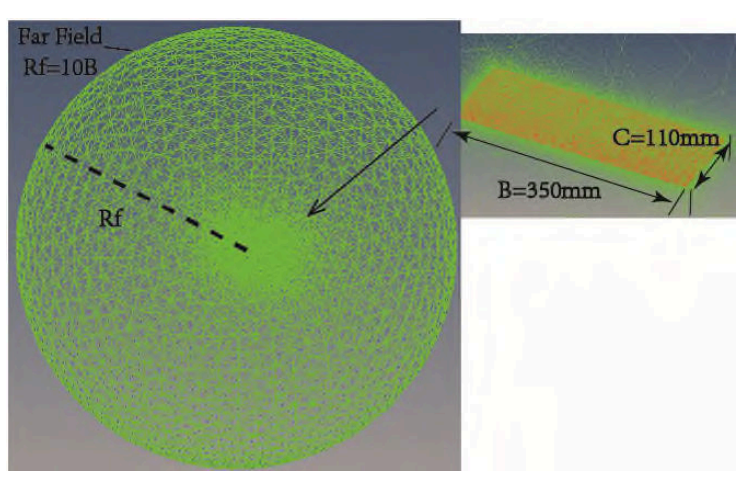

Figure 7. CFD model for the aerodynamic simulation of the flapping wing

The resulting lift coefficient $C_{L}$ and drag coefficient $C_{D}$ results in the range of $A o A=0 \sim 15$ degree are plotted in Error! Reference source not found.. The coefficients obtained by thin airfoil theory and lift line theory(Anderson 2001) (LLT) are also plotted for comparison purpose. The results shown in 
Error! Reference source not found. indicate that the $\mathrm{C}_{\mathrm{L}}$ by CFD become smaller than that by the LLT when $\mathrm{AoA}>9^{\circ}$. The tail-plane was modelled in the same CFD method as the wing.
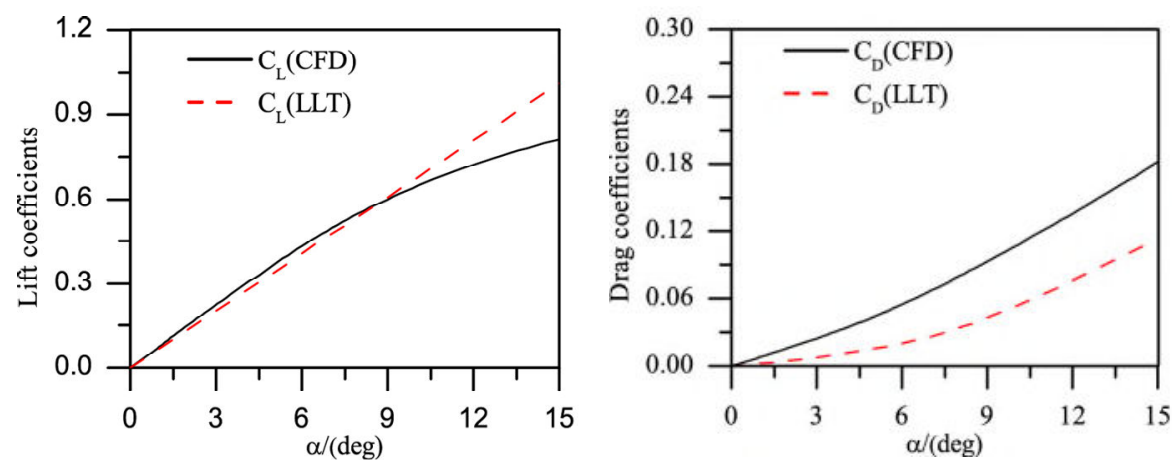

Figure 8. (a) Lift coefficient and (b) drag coefficients of the wing by CFD and LLT

Both wings were set in the mid of down-stroke position with an incidence angle $0^{\circ}$. The tail-plane was install in an angle $\alpha_{t w}=\alpha_{o g}=-2^{\circ}$ (trailing edge upward) relative to the body axis. Based on the above models and analysis, a level flight condition near ground was determined with aircraft pitching angle $\theta=7^{\circ}$, tail-plane deflection angle $\alpha_{o g}=-2^{\circ}$ and flight speed $\mathrm{V}=8.35 \mathrm{~m} / \mathrm{s}$. These resulting parameters were set in the flight dynamics model of the aircraft to simulate the stage-1 landing.

\section{Flight dynamic simulation of the aircraft short landing (stage 2 and 3)}

A flight dynamics model has been created by employing ADAMS to simulate the aircraft motion interfacing with the UAM for unsteady aerodynamic force calculation. The parameters during the landing include the tail-plane deflection angle $\alpha_{d e}$ starting from time $t_{d e}$ for a short period $d t_{d e}$ and the kinematics of motion, flapping frequency $f$ and start time $t_{f}$ of flapping wing. For all cases in the study, the kinematic of motion of the flapping wing is presented in Error! Reference source not found. where $\hat{t}$ is the non-dimensional time normalized by flapping period and $\hat{t}=\hat{t}_{f}$ when the flapping motion starts.

In this specified kinematics of flapping motion, the aircraft motion during landing was simulated with the parameters including tail-plane deflect angles varying in a range $\left(\alpha_{d e}=-10^{\circ},-20^{\circ},-30^{\circ}\right.$, $\left.-50^{\circ}\right)$, flapping frequencies $(f=0 \mathrm{~Hz}, 3 \mathrm{~Hz}, 4 \mathrm{~Hz})$ and starting time $\left(t_{f}=40.5 \mathrm{~s}, 41 \mathrm{~s}\right.$ or $\left.41.5 \mathrm{~s}\right)$ of the flapping wing motion. The simulation was started from the stage- 1 steady level flight at $V=8.35 \mathrm{~m} / \mathrm{s}$ near the ground for $40 \mathrm{~s}$ and followed by stage-2 landing with a transient $\alpha_{d e}$ for a short period $d t_{\text {end }}=$ $0.5 \mathrm{~s}$. The stage-3 landing was simulated in fixed wing mode $(f=0 \mathrm{~Hz})$ and flapping mode $(f=$ $3 \mathrm{~Hz}, 4 \mathrm{~Hz}$ ) starting from $t_{f}=40.5 \mathrm{~s}, 41 \mathrm{~s}$ or $41.5 \mathrm{~s}$ respectively until an ending time $t_{\text {end }}$. The safe landing 
condition was set as that the forward velocity was reduced from $8.35 \mathrm{~m} / \mathrm{s}$ to $V_{x}<1 \mathrm{~m} / \mathrm{s}$ and the descending velocity $V_{z}<2 \mathrm{~m} / \mathrm{s}$ in vertical direction within $2 \mathrm{~s}$.

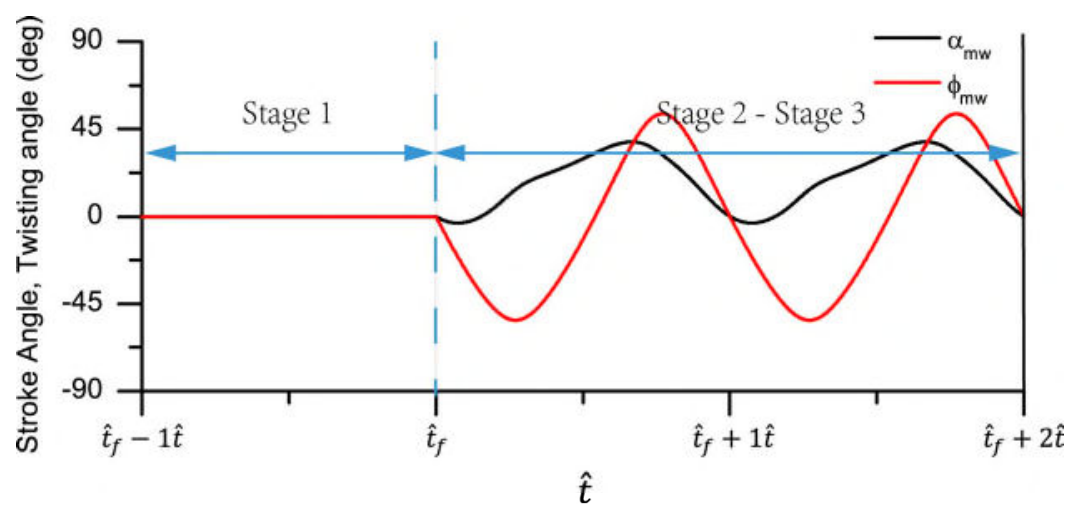

Figure 9. Flapping angle $\left(\phi_{m w}\right)$ and twist angle $\left(\alpha_{m w}\right)$ of the flapping wing relative to the body.

Error! Reference source not found. shows the results of the pitching angle of the aircraft (positive nose up), lifting force (positive upward) and drag (positive backward) produced by the wings during landing in three cases. After the level flight for 40s in stage-1 to approach landing, the tail-plane deflected at $t_{d e}=40 \mathrm{~s}$ to reach $\alpha_{d e}=-10^{\circ}$ within $0.5 \mathrm{~s}$ in the stage- 2 landing. The deflection caused an increase of the pitching angle $\theta$ and drag as shown in Error! Reference source not found.(a) and (c). In the same time, the lifting force was reduced for the first $0.15 \mathrm{~s}$ and then increased to a greater value than the level flight condition at the end of stage-1 as shown in Error! Reference source not found.(b). After $40.5 \mathrm{~s}$ in the stage-3 landing, if the wings remain fixed (fixed-wing mode $f=0 \mathrm{~Hz}$ ), the lifting force reduced as a result of continuing increase of the $\theta$ to maximum $28^{\circ}$ and drag for a short time and then reduced too. In this case, the aircraft landed like a glider without flapping and propulsion. This particular case (case 1-1) study results were taken as the reference to compare with the flapping wing performance. For the aircraft landing in flapping wing mode starting from $t_{f}=40.5 \mathrm{~s}$, two flapping cases were considered ( $f=3 \mathrm{~Hz}$ in case $1-2, f=4 \mathrm{~Hz}$ in case 1-3). The resulting $\theta$ oscillated and reached a peak value $\left(\theta=39.6^{\circ}\right.$ for $f=3 \mathrm{~Hz}, \theta=47.8^{\circ}$ for $\left.f=4 \mathrm{~Hz}\right)$ as shown in Error! Reference source not found. (stage 3 ). The unsteady aerodynamic lift and drag forces also varied. When the wing was in down-stroke, the lifting and drag forces increased as the flapping frequency increased; in upstroke, the lifting and drag forces decreased to small or negative values. 

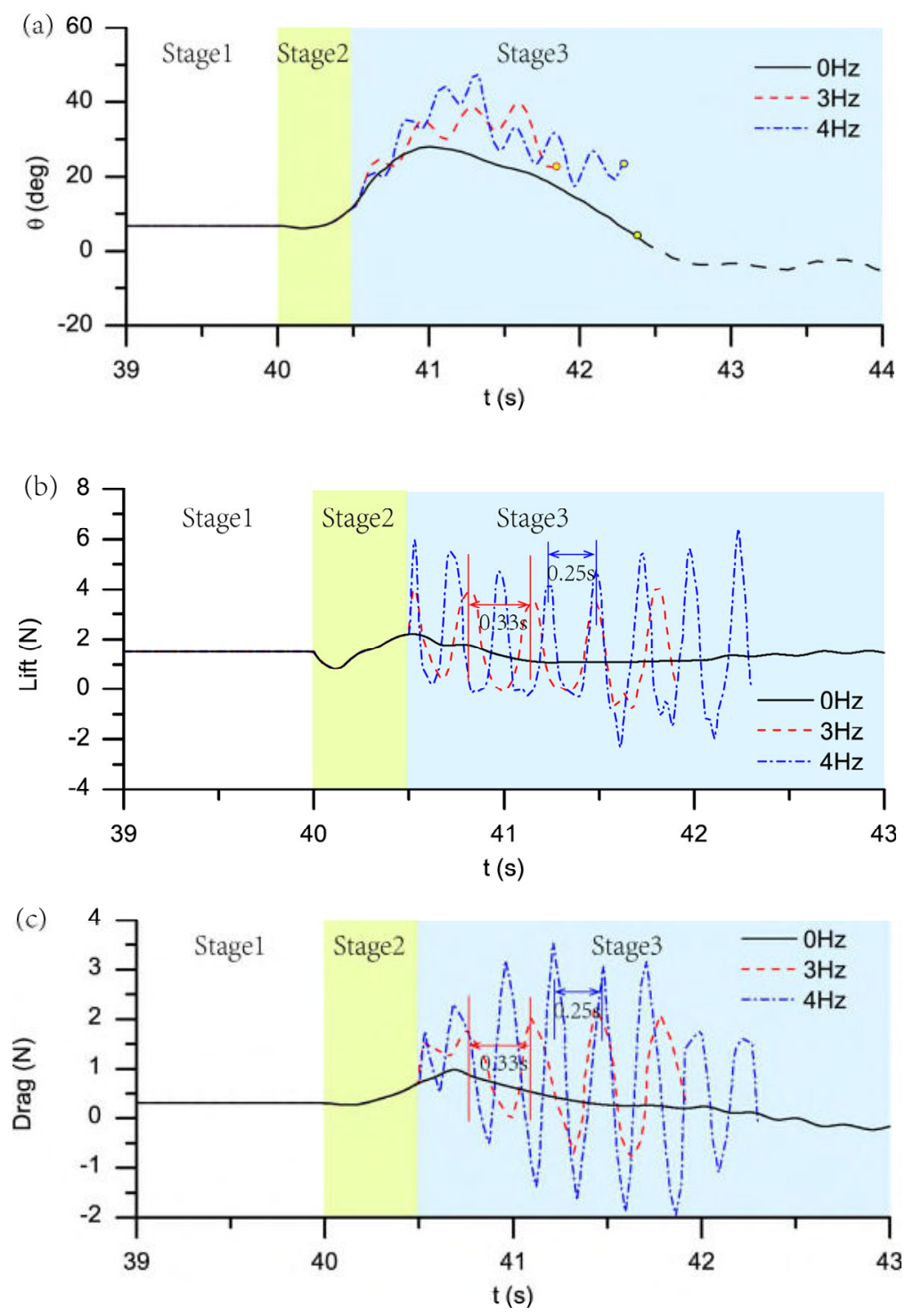

Figure 10. (a) Pitching angle of the aircraft (b) aerodynamic lifting force of both wings and (c) drag in response to $\alpha_{d e}=-10^{\circ}$ in fixed wing and flapping wing modes during landing

Corresponding to the above aerodynamic force and the inertia force of the aircraft, the resulting vertical and horizontal velocities of the aircraft during the landing are shown in Error! Reference source not found. As shown in Error! Reference source not found.(a), the vertical velocity $V_{z}$ (downward negative) of the aircraft increased from $t_{d e}=40 \mathrm{~s}$ for a short period and reached a maximum $V_{z}=-0.75 \mathrm{~m} / \mathrm{s}$ in response to $\alpha_{d e}=-10^{\circ}$, and then reduced back to zero at $41 \mathrm{~s}$ in the fixed wing mode (case 1-1). The results indicate that the aircraft descended from level flight position due to gravity and reduced lifting force, and then moved up again due to increased lift as shown in Error! Reference source not found.(b). Due to the increasing drag as shown in Error! Reference source not found.(c), the forward velocity $V_{x}$ of the aircraft started decreasing from stage-2 as shown in Error! Reference source not found.(b) and eventually reached minimum value $V_{x}=2.2 \mathrm{~m} / \mathrm{s}$ at $t=42.5 \mathrm{~s}$. In the same 
time, the aircraft moved downward again after $41 \mathrm{~s}$ and eventually reached $V_{z}=-3.12 \mathrm{~m} / \mathrm{s}$ at $t=42.5 \mathrm{~s}$ and approached a nearly stabilized status close to the maximum value. It is noted that the resulting velocities could not satisfy the short landing condition.
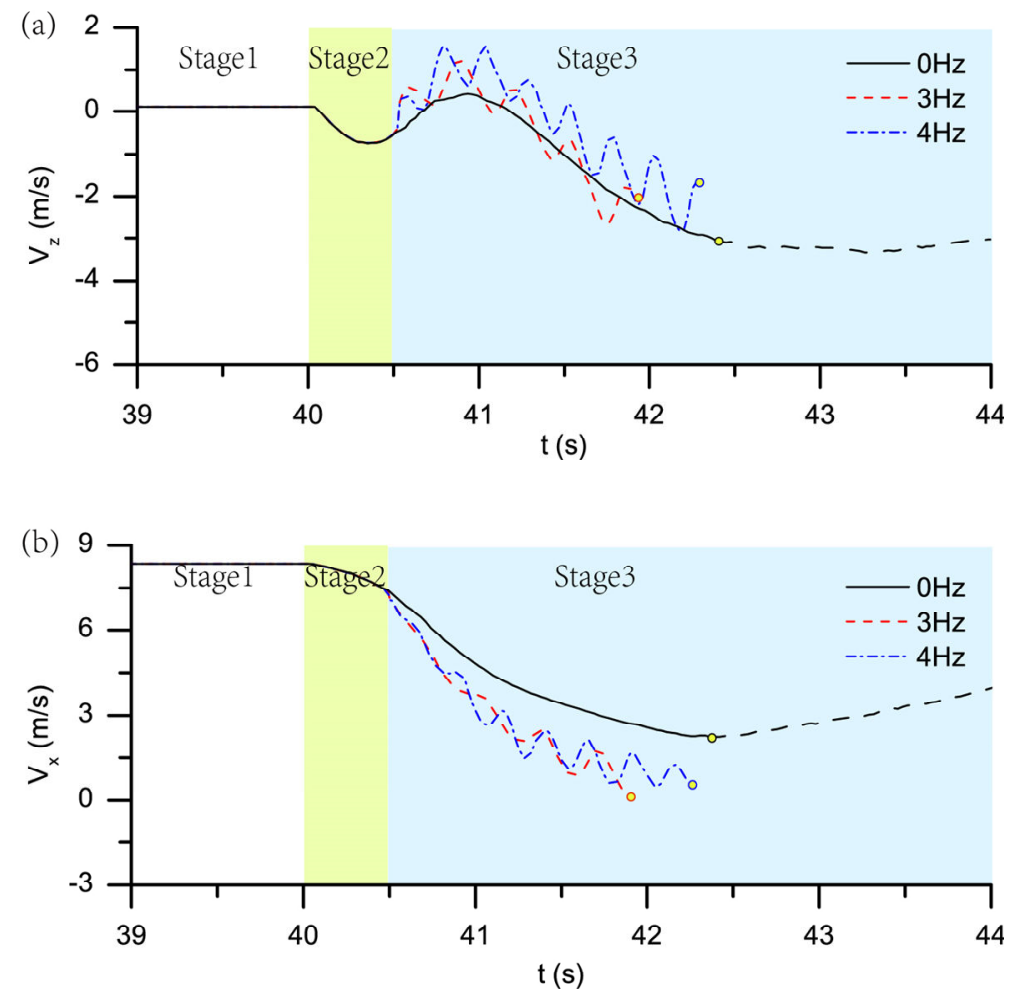

Figure 11. (a) vertical velocity (positive upward) (b) horizontal velocity (forward positive) of the aircraft in the ground coordinate system

When the flapping motion started from $t_{f}=40.5 \mathrm{~s}$ in stage-3 (case 1-2 $f=3 \mathrm{~Hz}$, case 1-3 $f=4 \mathrm{~Hz}$ ), the $V_{z}$ and $V_{x}$ of the aircraft oscillated corresponding to the aerodynamic forces as shown in Error! Reference source not found. When the flapping wing was in down-stroke in the period $t=$ $40.5 \mathrm{~s} \sim 42.5 \mathrm{~s}$, the aircraft reached $V_{z}=-2.09 \mathrm{~m} / \mathrm{s}$ and $-1.59 \mathrm{~m} / \mathrm{s}$ at $t_{\text {end }}$ for the two cases respectively. When the flapping wing was in up-stroke, the resulting $V_{z}<2 \mathrm{~m} / \mathrm{s}$ was in the same level as the fixed-wing mode (case 1-1) as shown in Error! Reference source not found.(b). In the same time, the $V_{x}$ was reduced to less than $2 \mathrm{~m} / \mathrm{s}$ at $t=42.3 \mathrm{~s}$ and zero at $t=41.9 \mathrm{~s}$ as shown in Error! Reference source not found.(b). It is noted that the resulting velocities by flapping wing mode are smaller than the fixed-wing and satisfy the short landing condition.

Consequently, the aircraft landing trajectory in terms of downward and forward displacement in the ground X-Z coordinate system is shown in Error! Reference source not found.. As shown in Error! Reference source not found.(a), the aircraft approached landing in stage- 1 at attitude $D_{z}=2 \mathrm{~m}$ and eventually descended to $D_{z}=-0.4 \mathrm{~m}, 1.2 \mathrm{~m}$ and $1.17 \mathrm{~m}$ measured from the ground level at $t_{\text {end }}$ in the 
case 1-1 $(f=0 \mathrm{~Hz})$, case 1-2 $(f=3 \mathrm{~Hz})$ and case 1-3 $(f=4 \mathrm{~Hz})$ respectively. Error! Reference source not found.(b) shows that the aircraft in fixed wing (case 1-1, $f=0 \mathrm{~Hz}$ ) moved from $D_{x}=8.4 \mathrm{~m}$ at $t=40 \mathrm{~s}$ further forward to $D_{x}=19.6 \mathrm{~m}$ to land at $t_{\text {end }}=42.5 \mathrm{~s}$. While for the flapping wing mode, the aircraft moved forward to $D_{x}=16.5 \mathrm{~m}$ in case $1-2(f=3 \mathrm{~Hz})$ and slightly further to $D_{x}=16.9 \mathrm{~m}$ in case 1-3 $(f=4 \mathrm{~Hz})$ at $t_{\text {end }}$ when $V_{x}=0 \mathrm{~m} / \mathrm{s}$, which is $3 \mathrm{~m}$ shorter than the fixed wing (case 1-1).
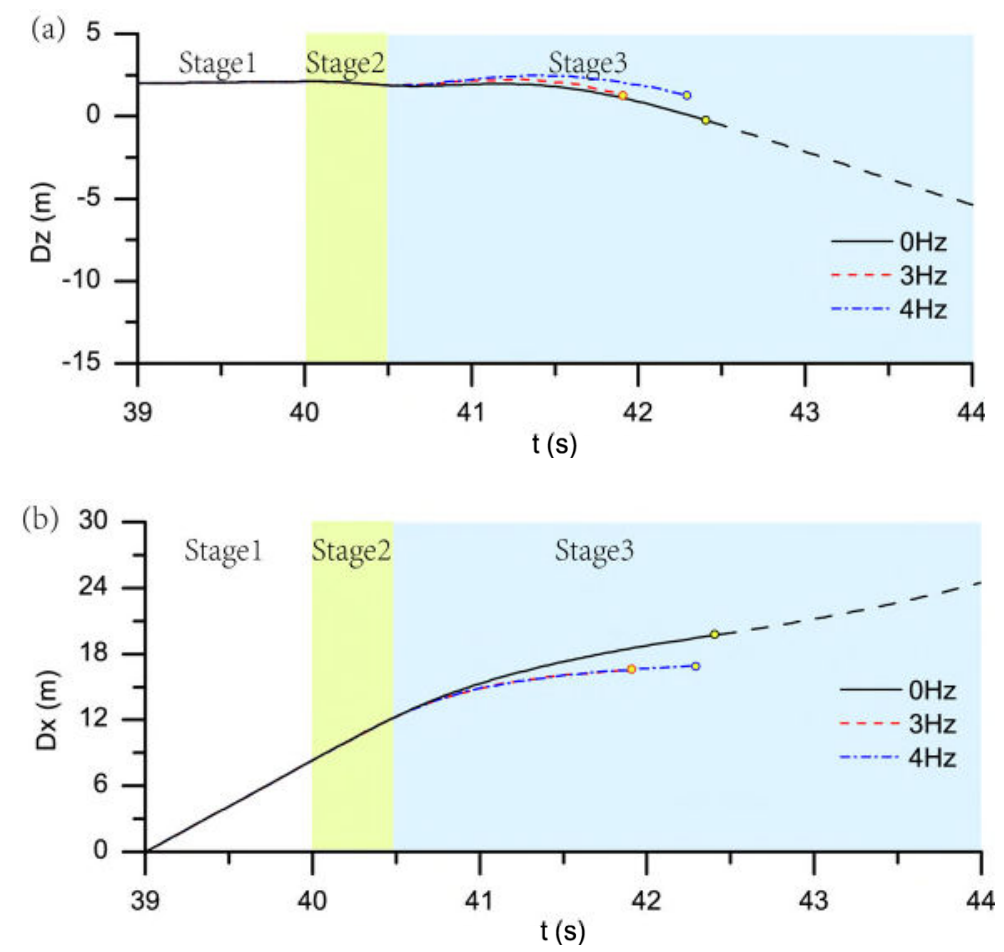

Figure 12. (a) Vertical movement of the aircraft in $\mathrm{Z}$ direction (positive for upward) (b) Horizontal movement in $\mathrm{X}$ direction (positive for forward) for $\alpha_{d e}=-10^{\circ}$

\section{Parametric study of the aircraft in short landing}

Based on the flight dynamics model, a parametric study has been carried out to evaluate the landing performance of the aircraft in an extended range of parameters $\alpha_{d e}\left(-10^{\circ},-20^{\circ},-30^{\circ},-50^{\circ}\right)$, $f(3 \mathrm{~Hz}, 4 \mathrm{~Hz})$ and $t_{f}(40.5 \mathrm{~s}, 41 \mathrm{~s}, 41.5 \mathrm{~s})$ including the above case-1. The resulting $V_{x}$ and $V_{z}$ corresponding to those operating parameters are listed in Error! Reference source not found.. For each of the tail-plane $\alpha_{d e}$, the results of the fixed-wing mode ( $f=0 \mathrm{~Hz}$ ) provide reference for comparison with the landing results of the flapping wing mode. For the fixed-wing mode, the resulting $V_{x}$ and $V_{z}$ normally do not satisfy the specified safe landing condition. In the flapping wing mode, the $V_{x}$ was reduced quickly in response to the increasing pitching angle and drag in landing as shown in Error! 
Reference source not found.. It is also noted that the flapping wing at higher frequency results in slightly larger forward velocity at the $t_{\text {end }}$ due to the associated thrust.

Table 3. The input parameters and landing analysis results until an ending $\boldsymbol{t}_{\boldsymbol{e n d}}$ when forward velocity is reduced to zero or the vertical velocity below $2 \mathrm{~m} / \mathrm{s}$

\begin{tabular}{|c|c|c|c|c|c|c|c|}
\hline Case No. & $\alpha_{d e}\left(^{\circ}\right)$ & $d t_{d e}(s)$ & $t_{f}(s)$ & $f(\boldsymbol{H z})$ & $t_{\text {end }}(s)$ & $V_{x}(m / s)$ & $V_{z}(m / s)$ \\
\hline $1-1$ & -10 & 0.5 & 40.5 & 0 & 42.40 & 2.23 & -3.06 \\
\hline $1-2$ & -10 & 0.5 & 40.5 & 3 & 41.92 & 0.00 & -2.09 \\
\hline $1-3$ & -10 & 0.5 & 40.5 & 4 & 42.30 & 0.27 & -1.59 \\
\hline $2-1$ & -20 & 0.5 & 40.5 & 0 & 42.3 & 1.33 & -3.21 \\
\hline $2-2$ & -20 & 0.5 & 40.5 & 3 & 41.5 & 0.00 & -2.4 \\
\hline $2-3$ & -20 & 0.5 & 40.5 & 4 & 41.5 & 0.2 & -1.62 \\
\hline $3-1$ & -30 & 0.5 & 40.5 & 0 & 41.64 & 0.00 & -3.4 \\
\hline $3-2$ & -30 & 0.5 & 40.5 & 3 & 41.48 & 0.00 & -2.9 \\
\hline $3-3$ & -30 & 0.5 & 40.5 & 4 & 41.50 & 0.22 & -1.67 \\
\hline $4-1$ & -50 & 0.5 & 40.5 & 0 & 41.25 & 0.00 & -4.1 \\
\hline $4-2$ & -50 & 0.5 & 40.5 & 3 & 41.16 & 0.00 & -2.96 \\
\hline $4-3$ & -50 & 0.5 & 40.5 & 4 & 41.26 & 0.00 & -1.92 \\
\hline $5-1$ & -10 & 0.5 & 41.0 & 0 & 42.40 & 2.23 & -3.06 \\
\hline $5-2$ & -10 & 0.5 & 41.0 & 3 & 42.14 & 0.00 & -2.32 \\
\hline $5-3$ & -10 & 0.5 & 41.0 & 4 & 42.08 & 0.68 & -1.68 \\
\hline $6-1$ & -20 & 0.5 & 41.0 & 0 & 42.3 & 1.33 & -3.21 \\
\hline $6-2$ & -20 & 0.5 & 41.0 & 3 & 42.4 & 0.07 & -2.68 \\
\hline $6-3$ & -20 & 0.5 & 41.0 & 4 & 42.1 & 0.42 & -1.85 \\
\hline $7-1$ & -30 & 0.5 & 41.0 & 0 & 41.64 & 0.00 & -3.4 \\
\hline $7-2$ & -30 & 0.5 & 41.0 & 3 & 41.60 & 0.00 & -3.07 \\
\hline $7-3$ & -30 & 0.5 & 41.0 & 4 & 41.82 & 0.00 & -2.05 \\
\hline $8-1$ & -50 & 0.5 & 41.0 & 0 & 41.25 & 0.00 & -4.1 \\
\hline $8-2$ & -50 & 0.5 & 41.0 & 3 & 41.32 & 0.00 & -3.38 \\
\hline $8-3$ & -50 & 0.5 & 41.0 & 4 & 41.28 & 0.00 & -2.74 \\
\hline $9-1$ & -10 & 0.5 & 41.5 & 0 & 42.40 & 2.23 & -3.06 \\
\hline $9-2$ & -10 & 0.5 & 41.5 & 3 & 42.53 & 0.67 & -2.64 \\
\hline $9-3$ & -10 & 0.5 & 41.5 & 4 & 42.54 & 1.06 & -1.77 \\
\hline $10-1$ & -20 & 0.5 & 41.5 & 0 & 42.3 & 1.33 & -3.21 \\
\hline $10-2$ & -20 & 0.5 & 41.5 & 3 & 42.3 & 0.30 & -2.92 \\
\hline $10-3$ & -20 & 0.5 & 41.5 & 4 & 42.1 & 0.74 & -2.31 \\
\hline $11-1$ & -30 & 0.5 & 41.5 & 0 & 41.64 & 0.00 & -3.4 \\
\hline $11-2$ & -30 & 0.5 & 41.5 & 3 & 41.54 & 0.00 & -3.09 \\
\hline $11-3$ & -30 & 0.5 & 41.5 & 4 & 41.54 & 0.00 & -2.85 \\
\hline
\end{tabular}

Further details of the landing results for all the cases listed in Error! Reference source not found. are presented in Error! Reference source not found.-15. Error! Reference source not found. shows the resulting pitching angle $\theta$ of the aircraft body (positive for nose-up) due to tail-plane deflection $\alpha_{d e}$ started at $40 \mathrm{~s}$ and followed by fixed wing and flapping wing motion started at $t_{f}=$ $40.5 s, 41.0 s$ and $41.5 s$ respectively as listed in Table 3 . For the cases $1 \sim 4$ when flapping motion starting at $t_{f}=40.5 \mathrm{~s}$ with different $\alpha_{d e}$, Error! Reference source not found.(a) shows that the 
resulting $\theta$ increases with $\alpha_{d e}$ and flapping frequency except the case $\alpha_{d e}=-50^{\circ}$. This is because such a large $\alpha_{d e}$ results in a maximum $\theta$ exceeded $70^{\circ}$ as shown in Error! Reference source not found.(a). In addition, the average pitching angle of the wing relative to the aircraft body is about $20^{\circ}$ based on the specified kinematics of motion of the flapping wing. This leads to the pitching angle of the wing approaching a maximum $90^{\circ}$ that makes the wing effectively stroke in a horizontal plane. Hence the $\theta$ is not sensitive to the flapping frequency anymore. When the flapping motion started later at $t_{f}=$ 41s and $41.5 \mathrm{~s}$ (cases 5 11), smaller deflection $\alpha_{d e} \leq-20^{\circ}$ would make the aircraft $\theta$ no sensitive to flapping frequency as shown in Error! Reference source not found.(b) and Error! Reference source not found.(c). It is because the $\theta$ reached the maximum value after $41 \mathrm{~s}$ in similar way to case $1 \sim 4$ as shown in Error! Reference source not found.(a). The flapping motion starting afterward can make the wing pitching angle reach maximum value more effectively than the case $1 \sim 4$.
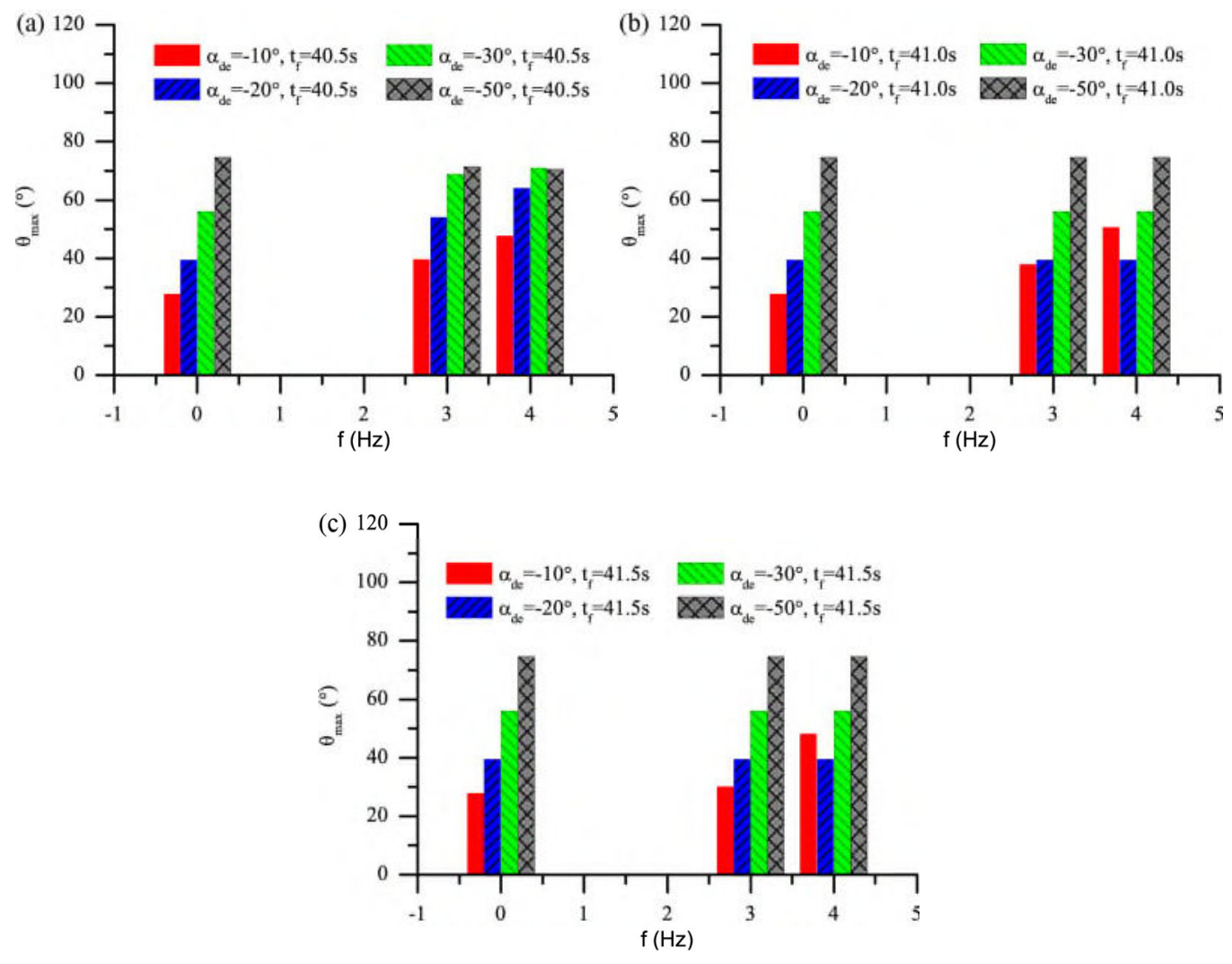

Figure 13. (a) Maximum pitching angle $\theta$ for $t_{f}=40.5 \mathrm{~s}$, (b) $t_{f}=41 \mathrm{~s}$ an (c) $t_{f}=41.5 \mathrm{~s}$ in all cases

As the results of the above cases, the descending velocity $V_{z}$ (negative for downward) of the aircraft in vertical direction at the ending time $t_{\text {end }}$ are shown in Error! Reference source not found.. For the fixed wing mode $(f=0 \mathrm{~Hz})$, the resulting $V_{z}$ of the aircraft increased with the $\alpha_{d e}$. It is because the fixed-wing would stall when the $\alpha_{d e}$ was beyond $-20^{\circ}$, hence the aircraft descended faster at the end 
of landing process. For the case $\alpha_{d e}=-10^{\circ}$ as example, the aircraft moved downward at $V_{z}=$ $-3.06 \mathrm{~m} / \mathrm{s}$ in $1.8 \mathrm{~s}$; for the case of increased $\alpha_{d e}=-50^{\circ}$, the aircraft descended faster at $V_{z}=$ $-4.2 \mathrm{~m} / \mathrm{s}$. When the flapping motion started at $t_{f}=40.5 \mathrm{~s}(f=3 \mathrm{~Hz}$, case 1-2) for example, the descending velocity was reduced to $V_{z}=-2.09 \mathrm{~m} / \mathrm{s}$ associated with $V_{x}=0 \mathrm{~m} / \mathrm{s}$ at $t_{\text {end }}=1.42 \mathrm{~s}$; for the higher $f=4 \mathrm{~Hz}$ (case $1-3$ ), the $V_{z}$ was further reduced to $-1.59 \mathrm{~m} / \mathrm{s}$ within $1.8 \mathrm{~s}$. It is noted that the time taken to reach the minimum $V_{z}$ in this case is the same as the fixed-wing mode. In the same range of $\alpha_{d e}$ and $f$ but a postponed flapping time at $t_{f}=41 \mathrm{~s}$ and $t_{f}=41.5 \mathrm{~s}$, the resulting $V_{z}$ is shown in Error! Reference source not found. (b) and Error! Reference source not found.(c). It is noted that the aircraft descended faster for larger $\alpha_{d e}$ especially for $\alpha_{d e}=-50^{\circ}$, and the variation trend is similar to the fixed wing case.
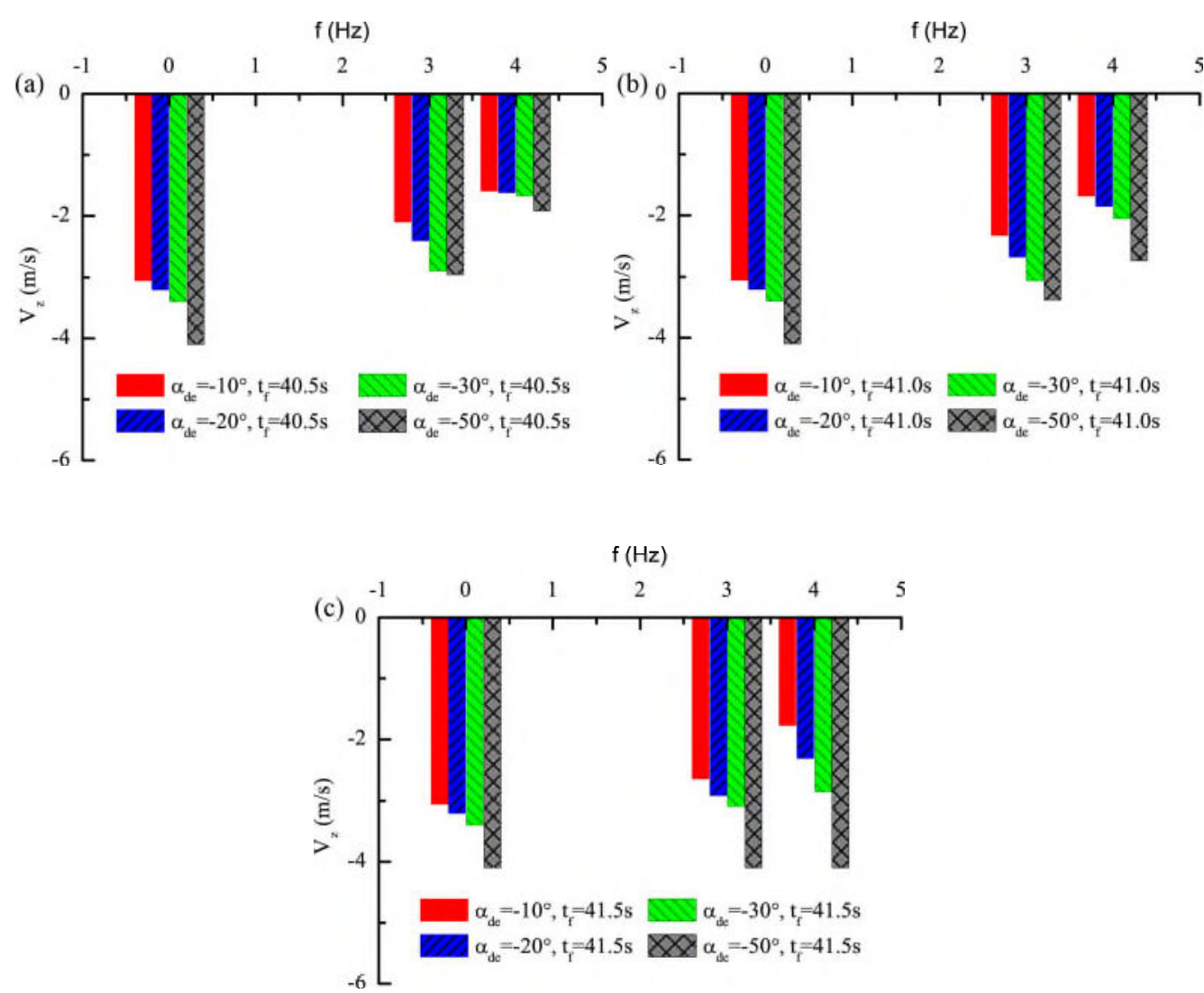

Figure 14. (a) Descending velocity $V_{z}$ (positive upward) at $t_{\text {end }}$ for $t_{f}=40.5 \mathrm{~s}$, (b) $t_{f}=41 \mathrm{~s}$ and (c) $t_{f}=41.5 \mathrm{~s}$.

Corresponding to the velocities in the landing process, the aircraft movement can be also measured in terms of displacement of the center of gravity (c.g.) in the X-Z plane of the ground coordinate system. In the fixed-wing case $(f=0 \mathrm{~Hz})$, the aircraft landing at larger $t_{\text {end }}$ results in larger forward displacement $D_{x}$ at $t_{\text {end }}$ as shown in Error! Reference source not found.(d). In the fixed-wing case 1- 
1 with a small $\alpha_{d e}=-10^{\circ}$, the aircraft landing behaves like a gliding process lasting for $1.9 \mathrm{~s}$ and results in a downward displacement $D_{z}=-0.4 \mathrm{~m}$ and longest forward movement distance $D_{x}=19.6 \mathrm{~m}$ at $t_{\text {end }}=42.4 \mathrm{~s}$. When the deflect angle is increased to $\alpha_{d e}=-30^{\circ}$ and $-50^{\circ}$, the forward movement distance is reduced to $D_{x}=14.9 \mathrm{~m}$ within $1.1 \mathrm{~s}$ and $D_{x}=13.49 \mathrm{~m}$ within $0.7 \mathrm{~s}$ respectively. In the flapping wing cases $1 \sim 4$, both the $D_{z}$ and $D_{x}$ are reduced significantly as the flapping frequency is increased. Taking the flapping wing case $3-3\left(f=4 \mathrm{~Hz}\right.$ and $\left.\alpha_{d e}=-30^{\circ}\right)$ as example, the forward movement distance is reduced to $D_{x}=14.33 \mathrm{~m}$ and downward displacement $D_{z}=1.6 \mathrm{~m}$. The results provide a reference for setting the aircraft altitude as a landing condition.
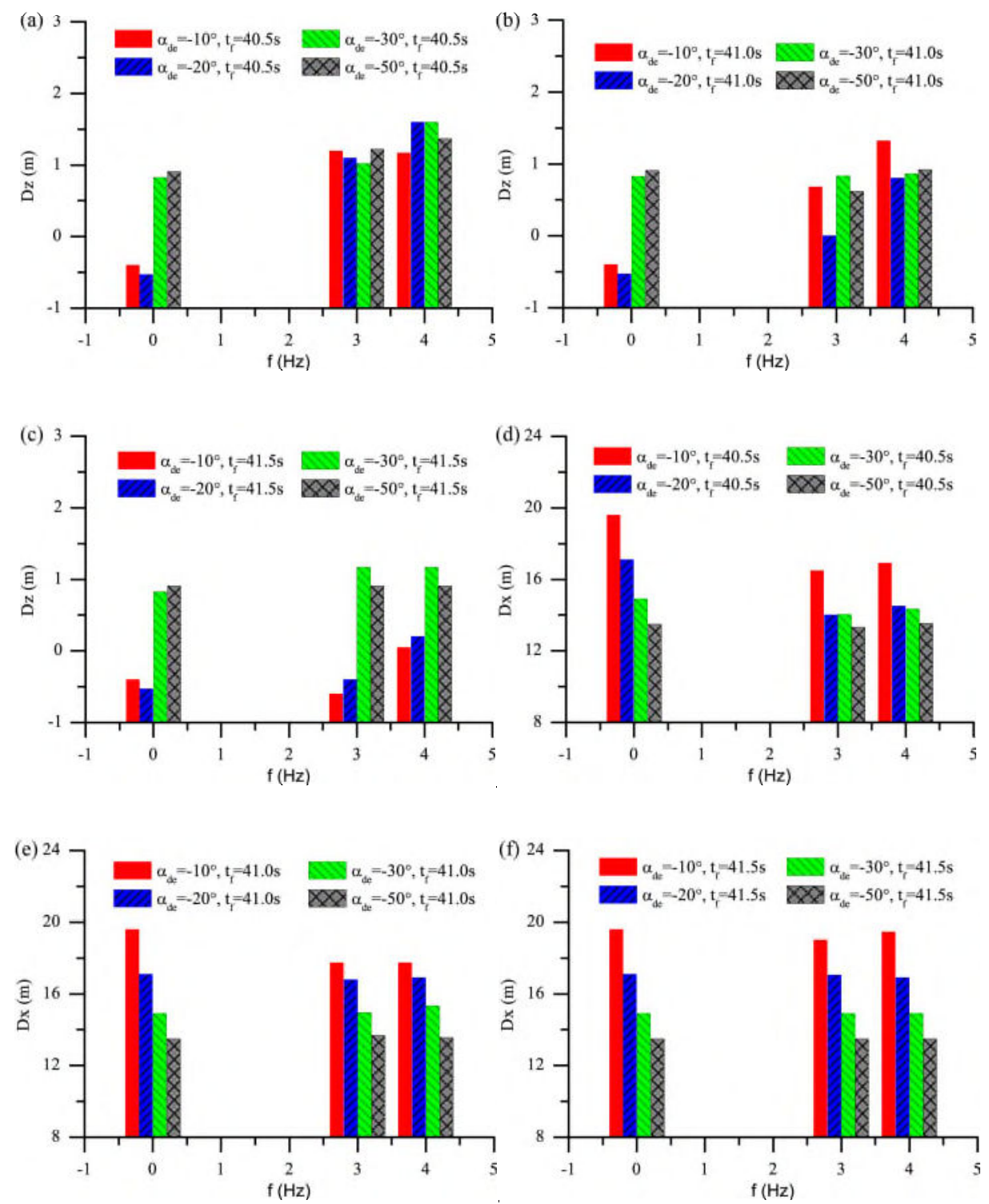

Figure 15. (a)(b)(c) Vertical displacements of the aircraft in landing (negative for downward movement), (d)(e)(f) Horizontal displacements in landing (positive for forward movement). 
The results provide a reference to set the aircraft altitude and flapping motion for short landing condition. Based on the above parametric study, the landing cases 1-3, 2-3 or case 3-3 are preferable to meet the landing condition of descending velocity below $2 \mathrm{~m} / \mathrm{s}$. The case $1-2$ is potentially an optimum landing scheme with smaller $\alpha_{d e}=-10^{\circ}$ and $f=3 \mathrm{~Hz}$ although the resulting descending velocity is slightly above $2 \mathrm{~m} / \mathrm{s}$.

\section{Scale effect on flapping wing aircraft short landing}

Since the flapping frequency is proportional to the mean lift (Guo et al. 2018; Zhou et al. 2014), which is related to the aircraft descending velocity in landing, a relationship between the flapping frequency and lift to weight ratio as a measure of the aircraft scale can be established. The lift to weight ratio can be expressed in equation (23), in which a quasi-steady aerodynamic force is adopted to represent the mean lift in a flapping cycle. Since the forward velocity $V_{x}$ will be reduced to a negligible small value at the end of landing process, only the flapping velocity $\left(2 \times \Phi \times f_{R F} \times R_{b}\right)$ due to the wing flapping motion is counted in the air velocity. According to the previous study (Chen et al. 2018; Gopalakrishnan and Tafti 2009; Wang et al. 2013) and the results shown in Error! Reference source not found., the mean lift coefficient of the flapping wing in such kinematic of motion in the $V_{x}=0$ condition is about $C_{L}=0.5$. Rearrange the equation (23), a required flapping frequency $f_{R F}$ to meet a specified descending velocity target can be determined from equation (24). The equation should be applicable to different geometric scale of aircraft and the required flapping frequency is normally inversely proportional to the scale.

$$
\begin{gathered}
L W R=\frac{1}{2} \times 1.225 \times C_{L} \times\left(2 \times \Phi \times f_{R F} \times R_{b}\right)^{2} \times S_{\text {total }} /\left(M_{\text {total }} g\right) \\
f_{R F}=\sqrt{L W R \times \frac{2 \times M_{\text {total }} g}{1.225 \times C_{L} \times S_{\text {total }}}} /\left(2 \times \Phi \times R_{b}\right)
\end{gathered}
$$

where $R_{b}$ and $S_{\text {total }}$ is the wing semi-span and total wing area respectively; $f_{R F}$ is the flapping frequency required to meet the specified descending velocity target in short landing.

The design target is to reduce the descending velocity $V_{z-f w}$ in flapping wing mode to a specified reduction factor relative to the descending velocity $V_{z-g}$ at $t_{\text {end }}$ in fixed-wing (gliding) mode as reference case. The reduction factor can be defined as $R F=1-V_{z-f w} / V_{z-g}$ to specify the target. For example, to achieve a target of reducing the $V_{z-f w}$ in flapping wing mode by $40 \%$ of the $V_{z-g}$ in landing, the $R F=0.4$. The $R F$ is increasing linearly with the specified reduction target, and also proportional to the lift to weight ratio $L W R$ expressed in equation (23). 
To verify the relationship and demonstrate the application of the equation (24), extended study is carried out for aircraft models of different total weight $0.0196 \mathrm{~kg}, 0.196 \mathrm{~kg}, 19.6 \mathrm{~kg}, 196 \mathrm{~kg}$ in a range weight scale $0.1,1,100,1000$ respectively. The weight scale is relative to the aircraft model studied in the 3 rd section as a baseline case $(0.196 \mathrm{~kg}$, weight scale 1$)$. Corresponding to the weight scales, the aircraft dimensions are also scaled in a set of geometric scale $0.5,1,5,10$ that lead to the aircraft design parameters. To achieve a target of $R F=0.5$ (reduce the descending velocity by $50 \%$ in flapping wing mode) as example, flight dynamics simulation for these scaled aircraft models in landing is performed. The same method as presented in the 3rd section is adapted in the simulation without taking the Reynolds number into account. To maintain the aircraft stability and determine the flight speed in the stage- 1 level flight, the tail-plane is set in a deflection angle $\alpha_{o g}=-2^{\circ}$. To initiate the stage- 2 landing process, the tail-plane angle is increased to $\alpha_{d e}=-10^{\circ}$ in a short period of $0.5 \mathrm{~s}$ (baseline model case 1). For each of the models, a flapping frequency $f_{R F}$ that meet the descending velocity target can be determined in the stage-3 simulation. Bring the resulting $f_{R F}$ value into equation (23), a $L W R$ can be calculated for each of the aircraft models. The obtained $f_{R F}$ and $L W R$ against the geometrical scale of the aircraft models is shown in Error! Reference source not found.. From the results, it is found that the $L W R$ keeps nearly constant with an average value of $L W R=0.51$ for the wide range of different scale aircraft. It is more important to note that the $L W R=0.51$ approximately equals to the specified descending velocity target $R F=0.5$. Given a scaled aircraft and a target $R F$ to replace the $L W R$ in equation (24), a required flapping frequency $f_{R F}$ can be determined as the key parameter for the flapping wing design and power estimation.

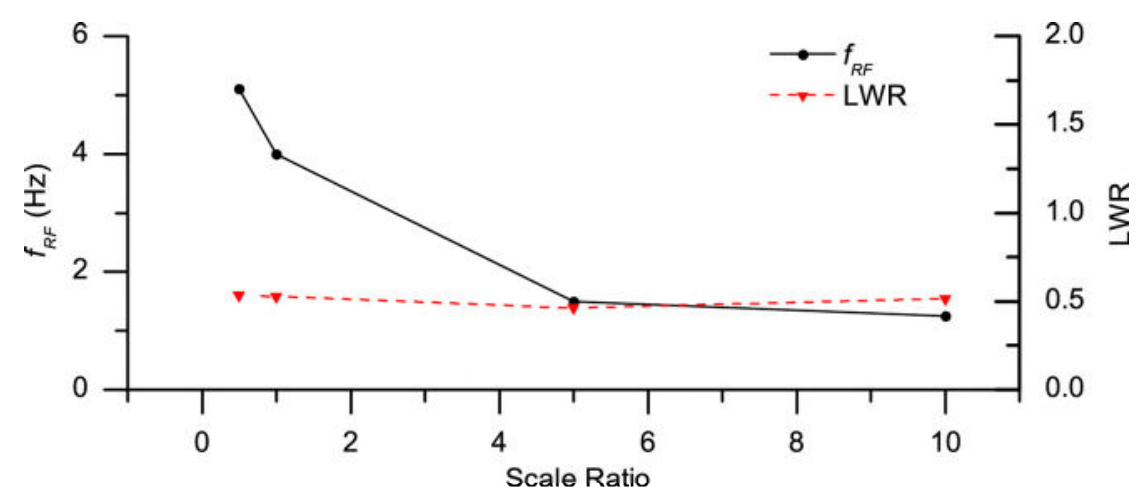

Figure 16. The relationship between $f_{50 \%}$ and the scale ratio of the flapping wing mechanism.

A flapping wing aircraft of scale ratio 10 of the same density as the original model (196kg in weight scale 1000) is selected to simulate the landing performance. A flight speed $V_{x}=30 \mathrm{~m} / \mathrm{s}$ with a tail-plane angle $\alpha_{o g}=-2^{\circ}$ was obtained to satisfy the level flight condition in the stage- 1 landing process. Since the large scale aircraft is of a greater mass moment of inertia than the small scale, a larger tail-plane 
deflection $\alpha_{d e}=-20^{\circ}$ was set to initiate the stage- 2 landing process at $t_{d e}=40 \mathrm{~s}$ for $0.5 \mathrm{~s}$. For the fixed-wing mode, the aircraft descended at $V_{z}=-2.5 \mathrm{~m} / \mathrm{s}$ at the end of stage- 2 as shown in Error! Reference source not found.(b). As a result of increasing $\theta$ in stage- 3 from $t=40.5 \mathrm{~s}$ as shown in Error! Reference source not found.(a), the $V_{z}$ was reduced back to zero and kept increasing to a positive value before reduced again. In the same time, the forward velocity of the aircraft was reduced continuously from $V_{x}=30 \mathrm{~m} / \mathrm{s}$ to a minimum $V_{x}=12 \mathrm{~m} / \mathrm{s}$ associated with $V_{z}=-8.5 \mathrm{~m} / \mathrm{s}$ at $t_{\text {end }}=$ $44 \mathrm{~s}$ as shown in Error! Reference source not found.(b) and (c). To achieve a short landing target $R F=$ 0.5 by flapping wing, the minimum flapping frequency needs to be determined to reduce the descending velocity to $\left|V_{z}\right|<4.25 \mathrm{~m} / \mathrm{s}$ within a time period of the fixed-wing mode. Thus a small range of flapping frequencies were attempted and the resulting $\theta, V_{z}$ and $V_{x}$ of the aircraft corresponding to $f=0.5 \mathrm{~Hz}$ and $1.25 \mathrm{~Hz}$ is presented in Error! Reference source not found.. It is noted that the aircraft motion is of similar trend to the small aircraft model (scale 1). For the lower $f=0.5 \mathrm{~Hz}$, the descending (vertical) velocity was increased to $V_{z}=-8.0 \mathrm{~m} / \mathrm{s}$ at $t_{\text {end }}=44 \mathrm{~s}$. The results show little difference from the fixed-wing mode although the forward (horizontal) velocity was reduced to $V_{x}=8.5 \mathrm{~m} / \mathrm{s}$, which is smaller than the fixed-wing mode. The results indicate that $f=0.5 \mathrm{~Hz}$ won't meet the specified landing target. For the $f=1.25 \mathrm{~Hz}$, the aircraft descending velocity is $V_{z}=-4.26 \mathrm{~m} / \mathrm{s}$ at $t_{\text {end }}=43.45 \mathrm{~s}$ as shown in Error! Reference source not found.(b), which has met the specified landing target. In the same time, the associated forward velocity was reduced to a minimum value $V_{x}=2.7 \mathrm{~m} / \mathrm{s}$ for a safe short landing.

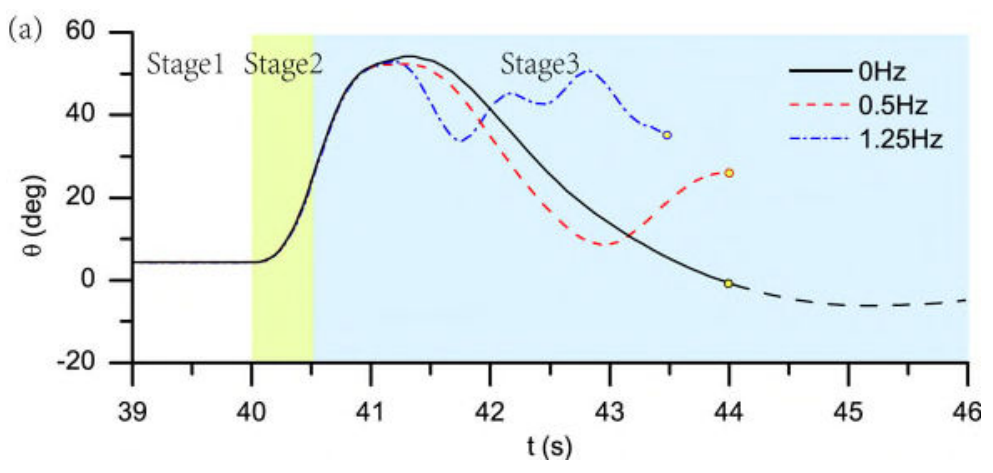



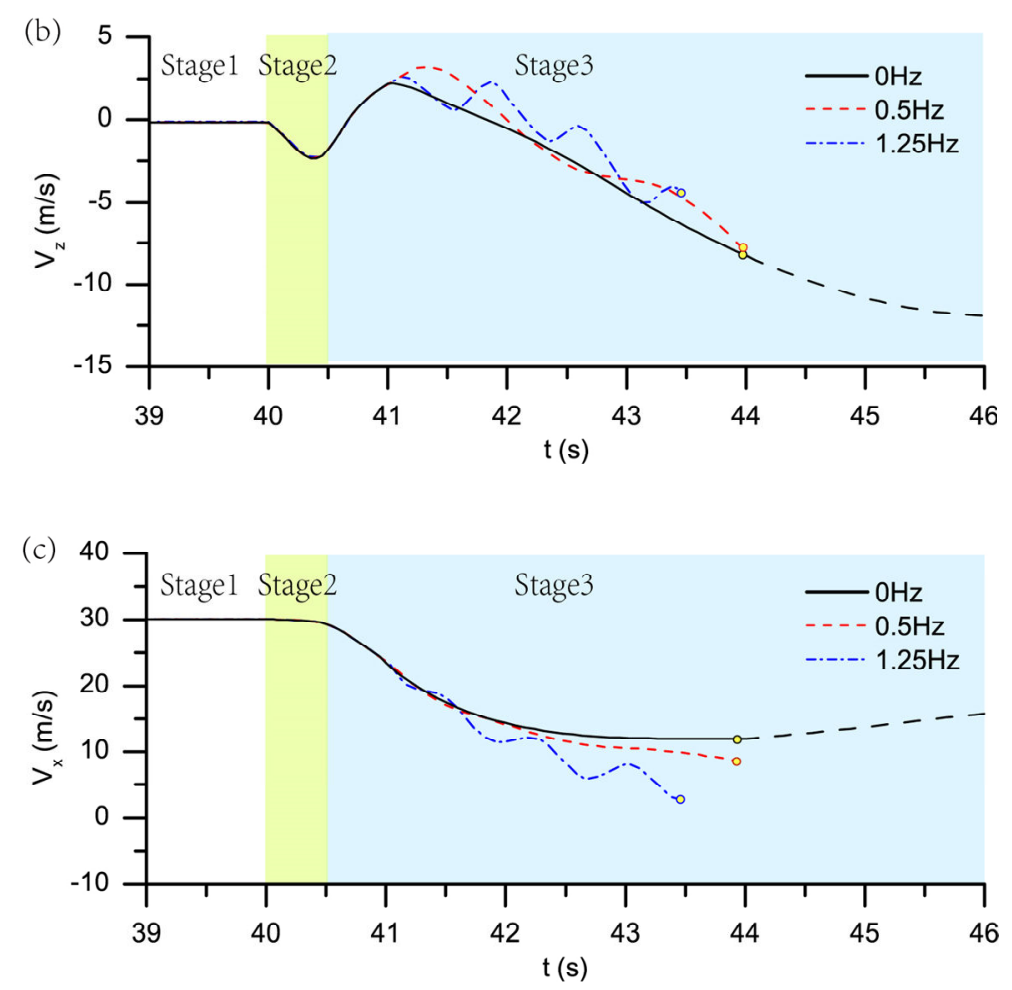

Figure 17. (a) Pitching angle of the aircraft (positive for nose-up) in response to $\alpha_{d e}=-20^{\circ}$ for $d t_{d e}=0.5 s$ (b) Vertical velocity (positive for upward) of the aircraft (c) Horizontal velocity (positive forward) of the aircraft in ground coordinate system (the dot marks indicate the $t_{\text {end }}$ ).

\section{Conclusion}

The present study has evaluated the effectiveness of flapping wing and scale effect on aircraft short landing. A flapping mechanism to transform a shaft rotation into a combined motion of flapping, twist and swing is designed to obtain an optimal kinematics of motion for a flapping wing. The effects of the vortex convection and shedding from the flapping wing leading edge and trailing edge are both taken into consideration in the aerodynamic analysis(Ansari et al. 2006b). The flight dynamics model of the aircraft takes into account the coupling between the inertia force and aerodynamic forces associated with the aircraft motion and flapping wing and tail-plane. To mimic the bird perching, the aircraft landing can be divided into three stages starting from a fixed wing mode at a velocity in level flight near ground. In stage 2, the short landing is then initiated by a transient deflection of the tail-plane to generate a quick increase of pitching angle of the aircraft. As a result, the forward velocity is reduced significantly. In the same time, the lifting force on the wing is increased for a short period before the wing stalls. After stall, the aircraft will descend with increasing velocity due to gravity. In the stage 3 , if the fixed wing mode is maintained, the aircraft will descend like a glider with both the forward and descending velocity 
reduced until landing. In the case of flapping wing mode, the aircraft forward and descending velocities vary in an oscillation manner. The reduction of descending velocity by flapping wing is proportional to the flapping frequency. Although the tail-plane deflection angle also affects the result, it does not play a dominant role.

Different scale of aircraft models has been studied in a rage of geometric scale $0.5,1,5$ and 10 corresponding to weight scale $0.1,1,100$ and 1000 respectively. A relationship is established between the lift to weight ratio and the flapping frequency required to reduce the aircraft descending velocity to a value relative to the fixed wing landing. In a range of the tail-plane deflection angle $-10^{\circ} \sim-20^{\circ}$, a minimum flapping frequency can be determined to achieve a specified target of descending velocity within a limited time. For example, a flapping frequency of $4 \mathrm{~Hz}$ is required for the aircraft of scale 1 to reduce the descending velocity by $50 \%$ of the same aircraft in fixed-wing mode. In the same time, the forward velocity is reduced to zero at $t_{\text {end }}$. For the large aircraft of scale 10, the required flapping frequency is $1.25 \mathrm{~Hz}$ to reduce the descending velocity by $50 \%$ of the fixed-wing mode. The method developed in the study can be used to determine the key design parameters for a flapping wing aircraft to achieve a specified short landing target.

\section{Data Availability Statement}

Some or all data, models, or code generated or used during the study are available from the corresponding author by request. (Including result data from Figure 1 to Figure 17).

Some or all data, models, or code generated or used during the study are proprietary or confidential in nature and may only be provided with restrictions. (Including the models or code used during the study).

\section{Acknowledgments}

The authors acknowledge the financial support from the National Key R\&D Program of China (2018YFB1305400) and China Scholarship Council (CSC No. 201606830010).

\section{References}

Akturk, A., Shavalikul, A., and Camci, C. (2009). "PIV Measurements and Computational Study of a 5-Inch Ducted Fan for V/STOL UAV Applications." 47th AIAA Aerospace Sciences Meeting. Anderson, J. D. J. (2001). Fundamentals of Aerodynamics. McGraw-Hill, New York.

Ansari, S. a., Zbikowski, R., and Knowles, K. (2006a). "Non-linear unsteady aerodynamic model for insect-like flapping wings in the hover. Part $1:$ methodology and analysis." Proc IMechE Part G: Journal of Aerospace Engineering, 220, 169-186. 
Ansari, S. a., Zbikowski, R., and Knowles, K. (2006b). "Non-linear unsteady aerodynamic model for insect-like flapping wings in the hover. Part 1 : methodology and analysis.” 220, 169-186.

Baligidad, S. M., Narayanaswamy, N., and Krishnamurthy, N. (2017). "Bio-inspired bi-plane flapping wing MAV." Open Journal of Mechanical Engineering( OJME ), 1(1), 4-7.

Berg, A. M., and Biewener, A. A. (2008). "Kinematics and power requirements of ascending and descending flight in the pigeon (Columba livia).” Journal of Experimental Biology, 211(7), $1120-1130$.

Chen, S., Li, H., Guo, S., Tong, M., and Ji, B. (2018). "Unsteady aerodynamic model of flexible flapping wing." Aerospace Science and Technology, Elsevier Masson SAS, 80, 354-367.

Deng, S., Percin, M., Van-Oudheusden, B., Remes, B., and Bijl, H. (2014). "Experimental Investigation on the Aerodynamics of a Bio-Inspired Flexible Flapping Wing Micro Air Vehicle.” International Journal of Micro Air Vehicles, 6(2), 105-115.

Englar, R. J., Hemmerlyt, R. A., Moore, W. H., Seredinsky, J. V., Valckenaere, W., and Jackson, J. A. (1981). "Design of the Circulation Control Wing STOL Demonstrator Aircraft." Journal of Aircraft, 18(1), 51-58.

Ghosh, S. K., Dora, C. L., and Das, D. (2012). "Unsteady Wake Characteristics of a Flapping Wing through 3D TR-PIV." Journal of Aerospace Engineering, 25(4), 547-558.

Gopalakrishnan, P., and Tafti, D. K. (2009). "Effect of Rotation Kinematics and Angle of Attack on Flapping Flight.” AIAA Journal, 47(11), 2505-2519.

Gordon, L. C. M. (1989). "Nonlinear Wake Evolution of Joukowski aerofoil in severe maneuver." Massachusetts Institute of Technology, Cambridge.

Graule, M. A., Chirarattananon, P., Fuller, S. B., Jafferis, N. T., Ma, K. Y., Spenko, M., Kornbluh, R., and Wood, R. J. (2016). "Perching and takeoff of a robotic insect on overhangs using switchable electrostatic adhesion." Science, 352, 978-982.

Guo, J. L., Chen, S. P., Li, L., Yuan, Z. Q., Wang, Y., and Ma, M. (2012). “An autonomously hopping-off micro raised-flapping-wing air vehicle." 31 st Chinese Control Conference, 4322 4327.

Guo, S., Li, H., Zhou, C., Zhang, Y. L., He, Y., and Wu, J. H. (2018). "Analysis and experiment of a bio-inspired flyable micro flapping wing rotor." Aerospace Science and Technology, 1, 1-13.

Jackowski, Z. J. (2009). "Design and Construction of an Autonomous Ornithopter." Massachusetts Institute of Technology, Cambridge.

Krashanitsa, R. Y., Silin, D., Shkarayev, S. V., and Abate, G. (2009). "Flight Dynamics of a FlappingWing Air Vehicle.” International Journal of Micro Air Vehicles, 1(1), 35-49. 
Martin, P., and Tung, C. (2004). "Performance and Flowfield Measurements on a 10-inch Ducted Rotor VTOL UAV." 60th Annual Forum of the American Helicopter Society.

Mishra, S., Tripathi, B., Garg, S., Kumar, A., and Kumar, P. (2015). "Design and Development of a Bio-Inspired Flapping Wing Type Micro Air Vehicle." International Conference on Nanomaterial's and Technologies, 519-526.

Montanya, J. B. D. L., and Marshall, D. D. (2007). "Circulation Control and Its Application to Extreme Short Take-Off and Landing Vehicles.” 45th AIAA Aerospace Sciences Meeting and Exhibit., 1-19.

Moore, J., Cory, R., and Tedrake, R. (2014). "Robust post-stall perching with a simple fixed-wing glider using LQR-Trees.” Bioinspiration and Biomimetics, 9(2), 025013.

Palmer, J. L., Jones, M. B., and Drobik, J. (2013). "Design elements of a bio-inspired micro air vehicle." IFAC Proceedings Volumes (IFAC-PapersOnline), 235-241.

Paranjape, A. A., Chung, S., Member, S., and Kim, J. (2013). "Novel Dihedral-Based Control of Flapping-Wing Aircraft With Application to Perching." IEEE Transactions on robotics, 29(5), 1071-1084.

Patra, A. K., Patel, K., Bundela, G. S., and Vinay, P. (2017). "Design and development of transition autopilot for VTOL UAV." International journal of innovations in engineering research and technology, 4(6), 57-72.

Prabu, G. M., Aravindhkumar, S. K., and Jegan, S. (2016). "Design Analysis and Fabrication of Delta Wing Amphibian UAV." International Journal of Engineering Research \& Technology, 5(04), 416-421.

Regan, W., Breugel, F. V., and Lipson, H. (2002). "Towards Evolvable Hovering Flight on a Physical Ornithopter." Proceedings of the Tenth International Conference on the Simulation and Synthesis of Living Systems, 241-247.

Roderick, W. R. T., Cutkosky, M. R., Lentink, D., and Roderick, W. R. T. (2017). “Touchdown to take-off : at the interface of flight and surface locomotion." Interface Focus, 7(1), 2016094.

Wang, D., Zhang, Y., Wu, J., and Zhang, Y. (2013). “Aerodynamics on Flapping Rotary Wing in Low Reynolds Number." American Institute of Aeronautics and Astronautics, 1-14.

Yang, W., Wang, L., and Song, B. (2018). "Dove: A biomimetic flapping-wing micro air vehicle." International Journal of Micro Air Vehicles, 10(1), 70-84.

Zhou, C., Wu, J., Guo, S., and Li, D. (2014). "Experimental study on the lift generated by a flapping rotary wing applied in a micro air vehicle." Proc IMechE Part G: Journal of Aerospace Engineering, 228(11), 2083-2093. 
2020-09-15

\section{Short landing performance and scale effect of a flapping wing aircraft}

Chen, $\mathrm{Si}$

ASCE

Chen S, Guo S, Li H, et al., (2020) Short landing performance and scale effect of a flapping wing aircraft. Journal of Aerospace Engineering, Volume 33, Issue 6, November 2020, Article number 0001198

https://doi.org/10.1061/(ASCE)AS.1943-5525.0001198

Downloaded from Cranfield Library Services E-Repository 